\title{
Development of Hovering type AUV "Cyclops" and its Performance Evaluation using Image Mosaicing
}

\author{
Juhyun $\mathrm{Pyo}^{\mathrm{a}}$, Hyeonwoo $\mathrm{Cho}^{\mathrm{a}}$, Hangil Joe ${ }^{\mathrm{a}}$, Tamaki Ura ${ }^{\mathrm{b}}$, Son-Cheol $\mathrm{Yu}^{\mathrm{a}, *}$ \\ ${ }^{a}$ Department of Creative IT Engineering, POSTECH, Pohang, 790-784, South Korea \\ ${ }^{b}$ Kyushu Institute of Technology, Fukuoka, Japan
}

\begin{abstract}
In this paper, a hovering-type autonomous underwater vehicle called Cyclops is introduced. Because of the symmetric body structure and thruster configuration of Cyclops, it is specially designed to utilize a lawnmower trajectory without changing its heading direction. This movement is effective at reducing the dead reckoning error and obtaining source images with homogeneous optical characteristics for underwater image mosaicing.

Keywords: autonomous underwater vehicle; underwater image mosaicing; underwater sensing; symmetric body structure; AUV design.
\end{abstract}

\section{Introduction}

Recently, the demand for underwater exploration has increased, with marine surveys being conducted for various purposes related to environmental issues, underwater resources, and scientific surveys (Yuh, 2000). These tasks commonly require the acquisition of underwater environmental data.

Autonomous underwater vehicles (AUVs) can potentially be applied to

\footnotetext{
* Corresponding author

Email address: sncyu@postech.ac.kr (Son-Cheol Yu)
} 
underwater sensing because they are capable of various underwater tasks: the inspection of underwater artificial structures (Kojima, 2003; Ura et al., 2005; Thornton et al., 2007), underwater intervention missions (Kim and Yuh, 2004; Marani et al., 2009), and exploration (Yuh, 1995; Kumagai et al., 2002; Griffiths, 2003; Bian et al., 2012).

AUVs can be divided into two categories: cruising and hovering types. Cruising-type AUVs are adequate for surveying large underwater areas over long distances (Hyakudome et al., 2002; Nasahashi et al., 2005). In contrast, hovering-type AUVs are suitable for missions that require precision ( $\mathrm{Li}$ et al., 2011; Whitcomb, 2009; Hayato et al., 2001; Ura et al., 2002; Hayato and Ura, 2004; Vaganay et al., 2006).

In this study, a hovering-type AUV called Cyclops was developed for sensing and scanning in shallow water. The body of Cyclops is symmetrical. Thus, it has the same hydrodynamic characteristics when moving forward and backward. As result, the modeling of the dynamics is simple. Therefore, its position and attitude can be controlled using a simple dual-loop proportional-integral-derivative (PID) controller. Its features make Cyclops suitable for moving in a lawnmower trajectory without changing its heading angle during a sensing mission.

Among the various underwater sensing methods, optical imaging is effective at perceiving underwater environment. However, only close-range underwater images are available because of the attenuation and backscattering of visible light in water (Sisman, 1982; Jaffe, 1990). This means that the field of view of underwater images is generally narrow. To extend the field of view, numerous source images are stitched together to form a high-resolution 
image that shows an area with a wide field of view (Pizarro and Singh, 2003; Ludvigsen et al., 2007). This approach is called image mosaicing (Szeliski, 1994; Faugeras and Luong, 2001).

However, underwater image mosaicing is challenging because of the insufficiently small overlap between adjacent images, perspective distortion, and nonhomogeneous optical conditions of the source images (Pizarro and Singh, 2003; Singh et al., 2004; Prados et al., 2012). Although many underwater image mosaicing algorithms have been proposed to solve these problems, and significant improvements have been achieved, the ultimate solution is obtaining appropriate source images that have a large overlap, no perspective distortion, and homogeneous optical conditions.

In this study, we performed underwater image mosaicing using Cyclops. For image mosaicing, Cyclops is controlled to move in a lawnmower trajectory and capture source images while maintaining its heading direction. This approach is effective at achieving homogeneous optical conditions for the source images. Therefore, the source images obtained by Cyclops satisfy the conditions needed for appropriate source images. In addition, maintaining the heading direction can reduce the complexity of the path planning for an image mosaicing mission. This task requires Cyclops to operate at its full capacity, and its sensing performance can be evaluated through the image mosaicing results.

\section{Development of Cyclops}

The purpose of our work is to develop a hovering type AUV that has accurate position control capability for underwater inspection such as image 
mosaicing, especially in real field. For this purpose, we considered all the design steps, including hardware implementation and control algorithm development. Cyclops has a symmetrical body structure (left-right, and frontrear) and symmetric thruster configurations, and hence, the AUV has similar hydrodynamics with respect to the surge, sway, and heave directions. Therefore, it can move in a lawnmower trajectory. Moreover, its movement with constant heading angle is effective in reducing the accumulation errors of the Gyro. This section explains the development of the hardware and software for Cyclops.

\subsection{Overview of Cyclops}

Cyclops is a small AUV that is sufficient for performing tasks in shallow water and coastal areas, as shown in Fig. 1. The specifications of Cyclops are summarized in Table 1.

The system configuration is shown in Fig. 2. Cyclops has two controllers: one is used for low-level control, which deals with sensor signal processing and thrust control, and the other is used for high-level control, which treats path planning and mission management. The low-level controller has a multifunctional signal I/O module and serial communication module. The multifunctional I/O module has 16 analog outputs and 24 digital I/O channels. The serial communication module has eight RS-232/RS-422/RS-485 channels, and is used for interfacing with various sensors. The controllers communicate with each other using the TCP/IP protocol. All of the sensors and controllers are powered by Li-Po batteries. 


\subsection{Hardware Design}

The hardware for Cyclops can be divided into the following categories: the main body frame, pressure housings, propulsion system, and external sensors. The main body of Cyclops is made of an aluminum frame, with an alumite coating applied to its surface at a thickness of more than $9 \mu \mathrm{m}$ (Misumi catalog, 2013). The body has a symmetrical shape for balance. Thus, Cyclops has the same hydrodynamic characteristics during forward and reverse movements, and left and right movements, which allows it to stably perform heave, sway, and surge motions. Moreover, the main frame of Cyclops is the bare-bones type. Therefore, all of the components, including the pressure housings, thrusters, and sensors, are directly installed on this frame, which makes it easy to change the system configuration. The layout of Cyclops is shown in Fig. 3.

One of the principal components of Cyclops is the main pressure housing, as shown in Fig. 4 (a). This pressure housing is composed of three parts: one connector junction housing, two cylindrical housings, and two acrylic dome housings. To ensure symmetricity, the front and rear ends have the same shape. The controllers, fiber-optic gyro, and thruster protector systems are securely installed inside the connector junction housing (see Fig. 4 (b)). The acrylic dome housings on the ends contain two pan/tilt cameras, as shown in Fig. 4 (c). The main pressure housing is mounted at the center of the Cyclops frame. In addition, two small pressure housings are installed at each side of the main frame. These housings also have symmetrical shapes similar to the main pressure housing. The battery system is located in these pressure housings (see Fig. 4 (d)). 


\subsection{Thruster Configuration}

Generally, a hovering-type AUV requires thrusters to maintain its position and attitude against disturbances. Using a few high-powered thrusters is one possible strategy, but precise control is difficult because of their highly nonlinear characteristics. Another strategy is using many low-powered thrusters. This is a more effective strategy for precise sensing applications. Therefore, the propulsion system of Cyclops is composed of eight identical thrusters, as shown in Fig. 5 (a) (Tecnadyne, 2014). The overall configuration of these thrusters is shown in Fig. 5 (b). Thrusters 1 and 2 are utilized for surge, and thrusters $3,4,5$, and 6 are used for sway motion. Thrusters 7 and 8 are used for heave motion. Because the drag force in the sway direction is higher than that of the others, half of the total number of thrusters is used for the sway motion. These thrusters allow Cyclops to run at a maximum speed of $2 \mathrm{kn}$.

\subsection{Sensor System}

The overall configuration of the sensors is shown in Fig. 6. The sensors of Cyclops can be categorized into navigation sensors and investigation sensors. The navigation sensors are used for feeding back the status of Cyclops to the control algorithm in real time. A Doppler velocity log (DVL) is used to measure the velocity and position, along with the surge and sway directions (Teledyne, 2014). In addition, a digital pressure transducer is used for depth measurement (Mensor, 2014). To measure the attitude of Cyclops, a high performance IMU is adopted (Honeywell, 2014). Although the IMU has drift

error, the amount of drift is acceptable for the following reason. Generally, the operation time of AUV is strictly restricted because of limited power 
supply such as that provided by an internal battery, while ROV has no such limitation because external power is supplied by its tether. For extra position sensors, a differential global positioning system is attached, and it can be utilized for positioning on the surface of the water (Novatel, 2014).

A forward-looking imaging sonar and profiling sonar are used as investigation sensors. The forward-looking imaging sonar is mounted at the bottom of Cyclops (Sound Metrics, 2014), and the profiling sonar is mounted on the top of Cyclops for scanning the surrounding environment (Imagenex, 2014). Cyclops also has optical camera systems. As previously mentioned, there are two pan/tilt cameras for real-time monitoring within the acrylic domes at the front and rear of Cyclops. The front of Cyclops is equipped with two LED lights whose brightness can be adjusted. A still-image camera and strobe light are mounted at the bottom of the frame and point in a downward direction. The still camera can take a color image with a resolution of 10 Mpixels and can be utilized for investigating the seafloor.

\subsection{Software Design}

Cyclops is controlled by its own software, which includes the automatic control algorithm. The software shows sensor information and the status of Cyclops in real time. Further, it provides an interface for manual operation, which is especially useful for emergency situations. To systemically supervise the components of Cyclops, we chose a hierarchical architecture because it is known to be an effective architecture for AUV control (Kim and Yuh, 2004). The graphic user interface of the software is shown in Fig. 7. Through this interface, operators can monitor the sensor information and status of Cyclops. In addition, Cyclops can be manually operated. 


\section{Proposed Strategy for Underwater Image Mosaicing}

Underwater image mosaicing is a useful technique for seafloor scanning. It requires image stitching, which is done by image registration and blending. The most significant problems that occur during the image stitching process are caused by the small area of overlap between two adjacent images, perspective distortion caused by the panning and tilting of the cameras, and non-homogeneous optical conditions (Pizarro and Singh, 2003; Singh et al., 2004; Prados et al., 2012).

Although these problems can be solved by means of advanced image processing algorithms, the ultimate solution is to obtain images that satisfy the following conditions: a large overlap, no perspective distortion, and homogeneous optical conditions. Under these conditions, underwater image mosaicing can be easily accomplished without the help of complicated image processing algorithms. Because Cyclops is especially designed for precise sensing and scanning, we can obtain the appropriate source images. In the following sections, the operating strategy for underwater image mosaicing by Cyclops is described in detail.

\subsection{Image Mosaicing Scenario}

When the seafloor area for image mosaicing is defined, it can be divided into a mesh grid. Then, waypoints are defined on the grid area. Finally, a path that connects all of these waypoints is generated. Cyclops captures the source images at every waypoint while sweeping the grid area by following the path. Finally, a panoramic image can be generated by stitching together the source images. 
To determine the density of the grid, the visible distance between the camera and the seafloor must be found. To overcome the turbidity of the water, the camera must approach the seafloor. Moreover, the distance has to be shortened to enhance the resolution of the panoramic image. If the distance $Z$ is specified, the width $W$ and height $H$ of the camera's field of view can be calculated as follows (Caccia, 2007): $W=2 Z \tan (\alpha)$ and $H=2 Z \tan (\beta)$, where $\alpha$ and $\beta$ are the camera's horizontal and vertical angles of view, respectively. For image mosaicing, adjacent source images must overlap each other. Therefore, we shrink the field of view using $W_{g}=S \times W$ and $H_{g}=S \times H$, where $S$ is the safety factor for image mosaicing and $0<S<1$. Finally, $W_{g}$ and $H_{g}$ can be used for the width and height of an element of the mesh grid, respectively, as shown in Fig. 8. The centers of the grid elements are set to the waypoints.

Next, the position error margin has to be established. We define the position error margin as a rectangular cuboid area whose center is the waypoint. Both the width and length of the cuboid are defined as $r_{p}$, and the height is given by $r_{h}$, as shown in Fig. 9. Once Cyclops is located within the cuboid area, a source image is taken by the camera. The position error margin and control effort for precise positioning have a trade-off relationship between the accuracy and elapsed time for underwater image mosaicing.

Navigation and control methods are required to achieve this scenario. Dead reckoning is used for navigation, which makes Cyclops track the adjacent waypoints on the grid area. The purpose of the control algorithm is to allow Cyclops to approach each waypoint as closely as possible. In the following sections, both methods are described. 


\subsection{Navigation Strategy}

The path of Cyclops must touch all the waypoints. Thus, we propose a lawnmower trajectory that connects all the waypoints. Cyclops must obtain the source images under homogeneous optical conditions and without perspective distortion, and these requirements are mainly related to its attitude.

To achieve homogeneous optical conditions for the images, Cyclops must not change its heading angle during path tracking. This means that the initial value of its yaw angle must be maintained. The constant heading is adopted to reduce dead-reckoning error as much as possible. Practically, frequent changes in heading angle can cause higher accumulation error. Moreover, the constant heading was needed to circumvent calculations for rotating images during image consolidation (Marks et al., 1994a,b, 1995; Fleischer et al., 1997). The lawnmower trajectory consists of several rows. When Cyclops reaches the last waypoint of a row, it moves to the next row. For this motion, Cyclops turns its heading angle by $90^{\circ}$, and then approaches the next row as shown in Fig. 10 (a). After Cyclops arrives at the next row, it turns its heading angle by $90^{\circ}$ again, and then continuous the operation. Because its camera and strobe light are mounted vertically on the right and left sides, respectively, the change in the heading angle causes a rotation of the source image. Therefore, the image stitching algorithm must rotate the images in opposite directions when stitching them to the corresponding images taken in the previous row. Moreover, the directions of the shadows on adjacent images are different, which causes a feature matching error between the images. As a result, complex image stitching algorithms should be used, and this strategy is not suitable for the proposed concept of underwater image mosaicing. 
An alternative strategy is shown in Fig. 10 (b). When Cyclops reaches the last waypoint of the row, it moves its sway direction without changing its heading angle to move to the next row of waypoints. In this case, there is no image rotation and no difference between shadows. This movement is possible because Cyclops uses individual thrusters for sway motion. In addition, the roll and pitch angles of Cyclops must also be fixed during operation to avoid perspective distortion. Cyclops is hydrodynamically stable in roll and pitch because of a buoyancy sheet at the top and the battery housing at the bottom. Therefore, the roll and pitch angles are readily controlled, and the angle of the camera remains stable.

Moreover, the proposed strategy of maintaining the heading direction is useful for aligning the camera with each waypoint. Generally, the camera is not mounted at the center of an AUV. Instead, a navigation sensor such as a DVL is located at the center, because the center of the AUV is treated as the reference point for AUV positioning. As shown in Fig. 11 (a), the relative position between the camera and each waypoint is varied while changing the heading direction. Therefore, the position of Cyclops must stray some distance from the waypoint to maintain the relative position after changing the heading direction. This makes the navigation scenario complex. In contrast, in Fig. 11 (b), the relative positions are identical at all of the waypoints. Therefore, Cyclops can capture source images at every waypoint without compensating for changes in the relative position.

In addition, the proposed strategy is effective at reducing dead reckoning error. Dead reckoning suffers from positioning error accumulation. Generally, a high positioning error is caused by changing the heading direction. In the 
proposed strategy, the heading direction of Cyclops is fixed, which mitigates the dead reckoning error. Furthermore, the proposed strategy is also useful for image mosaicing in a narrow space where the AUV cannot turn its body.

\subsection{Control Algorithm}

Body-fixed and earth-fixed coordinate systems are used to describe the motion of Cyclops, as shown in Fig. 12. The body-fixed coordinate system coincides with Cyclops. Therefore, its motion can be expressed as movements of the body-fixed coordinate system with respect to the earth-fixed coordinate system. The position of Cyclops is defined as $x, y$, and $z$, and its attitude is denoted as $\phi, \theta$, and $\psi$ with respect to the earth-fixed coordinate system, where $\phi, \theta$, and $\psi$ mean the roll, pitch and yaw angles, respectively. Moreover, the linear and angular velocities of Cyclops are expressed as $u, v$, $w, p, q$, and $r$ in the body-fixed coordinate system, as shown in Fig. 12. The relationships among these parameters are given as follows (Fossen, 1994):

$$
\begin{aligned}
& \dot{x}=u \cos \psi-v \sin \psi \\
& \dot{y}=u \sin \psi+v \cos \psi \\
& \dot{z}=w \\
& \dot{\psi}=r .
\end{aligned}
$$

In most cases, when controlling the AUV, the roll and pitch angles are assumed to be stable. Hence, $\phi$ and $\theta$ can be neglected. Then, the hydrodynamic model of Cyclops can be expressed as follows: (Fossen, 1994; Caccia 
and Veruggio, 2000)

$$
\begin{aligned}
m_{u} \dot{u} & =m_{v} v r-k_{u} u-k_{u|u|} u|u|+F_{u} \\
m_{v} \dot{v} & =m_{u} u r-k_{v} v-k_{v|v|} v|v|+F_{v} \\
m_{w} \dot{w} & =-k_{w} w-k_{w|w|} w|w|+F_{w}+W \\
I_{r} \dot{r} & =-\left(m_{v}-m_{u}\right) u r-k_{r} r-k_{r|r|} r|r|+T_{r},
\end{aligned}
$$

where $m_{u}, m_{v}$, and $m_{w}$ are the summations of the mass of Cyclops and the hydrodynamic masses in the $u, v$, and $w$ directions, respectively. $I_{r}$ is the moment of inertia corresponding to the rotation with respect to the $r$-axis. $k_{u}, k_{u|u|}, k_{v}, k_{v|v|}, k_{w}, k_{w|w|}, k_{r}$, and $k_{r|r|}$ are the linear and quadratic damping coefficients (Fossen, 1994). $F_{u}, F_{v}$, and $F_{w}$ are the forces exerted in the $u, v$, and $w$ directions, respectively. $T_{r}$ is the torque around the $r$-axis.

Moreover, the thrusters of Cyclops are calibrated to eliminate the nonlinear characteristics. The nonlinear characteristics of the thrusters are modeled as follows (Kim et al., 2013):

$$
u_{i}= \begin{cases}c_{1 i-} v_{i}^{2}+c_{2 i-} v_{i}+c_{3 i-}, & \text { if } v_{i} \leq v_{t h-} \\ 0, & \text { if } v_{t h-}<v_{i} \leq v_{t h+} \\ c_{1 i+} v_{i}^{2}+c_{2 i+} v_{i}+c_{3 i+}, & \text { if } v_{i}>v_{t h+},\end{cases}
$$

where $u_{i}$ is the propulsion of the $i$-th thruster, and $v_{i}$ is the input voltage of the thruster. $c_{1 i-}, c_{2 i-}, c_{3 i_{-}}, c_{1 i+}, c_{2 i+}$, and $c_{3 i+}$ are the model parameters of the thrusters. A thruster cannot generate propulsion if the voltage does not exceed the threshold voltage, i.e., $v_{t h-}$ or $v_{t h+}$. Therefore, dead zone exists where $v_{t h-}<v_{i} \leq v_{t h+}$. If the input voltage exceeds the threshold voltage, the thruster generates a propulsion that increases quadratically. 
The thrusters were calibrated based on the results of experiments. The calibration result for thruster 1 is shown in Fig. 13. The coefficients of the quadratic equation were derived using a regression analysis. All of the coefficients are listed in Table 2. Values of -1 and $1 \mathrm{~V}$ are given as the threshold voltages $v_{t h-}$ and $v_{t h+}$ for all the thrusters.

We control Cyclops using a dual-loop PID controller, as shown in Fig. 14. The inner-loop controller stabilizes the dynamics of Cyclops. The outer-loop controller regulates its position and attitude to the desired values. For the inner-loop controller, the velocity of Cyclops is fed back, and the velocity error is calculated by subtracting the desired velocity. The velocity error is defined as $e_{i}=\bar{V}-V$, where $\bar{V}$ and $V$ are the desired and current velocities of Cyclops, respectively. Based on the error, the inner-loop controller generates force $F$ as a control input using the following PID controller:

$$
F(t)=K_{P i} e_{i}(t)+K_{D i} \frac{d}{d t} e_{i}(t)+K_{I i} \int_{0}^{t} e_{1}(\tau) d \tau,
$$

where $F$ can be assigned to $F_{u}, F_{v}, F_{w}$, and $T_{r}$. The outer-loop PID controller regulates the position and attitude of Cyclops. The current position and attitude are fed back, and the error of the position and attitude is calculated as $e_{o}=R-\bar{P}$, where $R$ is the reference position and attitude, and $\bar{P}$ is the current position and attitude. The outer-loop PID controller is given by

$$
V(t)=K_{P o} e_{o}(t)+K_{D o} \frac{d}{d t} e_{o}(t)+K_{I o} \int_{0}^{t} e_{2}(\tau) d \tau,
$$

where $V(t)$ is the control velocity, and can be assigned to $u, v, w$, and $r$.

The generated forces and torque are realized by the propulsion from the thrusters. The number of thrusters is larger than the number of control forces because these can be generated by multiple thrusters. To assign the thrusters 
for generating the forces and torque, the following optimization criteria are used (Kim et al., 2013):

$$
\min _{\mathbf{u}} J=\frac{1}{2} \mathbf{u}^{T} \mathbf{W u}, \text { subject to } \mathbf{F}=\mathbf{B u}
$$

where $\mathbf{u}$ and $\mathbf{F}$ are defined as $\left[\begin{array}{llll}u_{1} & u_{2} & \cdots & u_{8}\end{array}\right]^{T}$ and $\left[\begin{array}{llll}F_{u} & F_{v} & F_{w} & T_{r}\end{array}\right]^{T}$, respectively. $\mathbf{W}$ is the weight matrix, and $\mathbf{B}$ defines the contribution of each thruster to $F_{u}, F_{v}, F_{w}$, and $T_{r}$. In this paper, $\mathbf{W}$ is given by an identity matrix and

$$
\mathbf{B}=\left[\begin{array}{cccccccc}
1 & 1 & 0 & 0 & 0 & 0 & 0 & 0 \\
0 & 0 & 1 & 1 & -1 & -1 & 0 & 0 \\
0 & 0 & 0 & 0 & 0 & 0 & 1 & 1 \\
0 & 0 & r \sin (\theta) & -r \sin (\theta) & -r \sin (\theta) & r \sin (\theta) & 0 & 0
\end{array}\right] .
$$

\section{Experimental Results}

\subsection{Control Accuracy}

The performance of the proposed controller was tested in a path-tracking experiment. This experiment was conducted in an indoor water tank, as shown in Fig. 15. The PID gains applied to the experiment are listed in Table 3 .

Nine waypoints were defined for the experiment, and a path was generated that connected all of the waypoints, as shown in Fig. 16. During the experiment, the heading of Cyclops was controlled to be constant. The blue line labeled target represents the path. The red line labeled trajectory(Fig. 16) is the moving trajectory of Cyclops in the experiment. Comparisons between the desired path and actual trajectory of Cyclops were shown in Fig. 17. On the whole experiment results, Cyclops tracked the desired path well, 
but there was small overshoot in each waypoint. However, the errors were attenuated over time, and the movement of Cyclops became stable.

\subsection{Mosaicing Test}

An image mosaicing experiment was conducted in other indoor water tank, as shown in Fig. 18 (a). In this mosaicing experiment, a specially designed printed cotton sheet was used to evaluate the accuracy of the image mosaicing, as shown in Fig. 18 (b). The size of this sheet was $3 \times 3$ $m$. Grid patterns and numerical characters were printed on the sheet. The accuracy of the image mosaicing could be readily evaluated by checking the connectivity of the patterns and characters at the seam lines of the mosaic image. The cotton sheet was evenly laid on the bottom of the water tank for the experiment.

To generate the mosaic image of the sheet, a square $4 \times 4 m$ area was defined as the target domain for image mosaicing. A total of 81 waypoints were assigned in the square area, and the gaps between adjacent waypoints were set to $0.5 \mathrm{~m}$, as shown in Fig. 18 (c). The distance between the camera and the bottom of the water tank was set to $1.8 \mathrm{~m}$.

Two image mosaicing experiments were conducted under these experimental setup. In the first experiment, the heading of Cyclops was fixed during navigation. In the second experiment, the heading was changed when Cyclops reached edge of the target domain. Dead reckoning based on a DVL and gyro was used for navigation.

A gyro was used only for the constant heading navigation. Generally, the magnetic compass can be also used to control the heading angle. The compass does not have drift errors, because it is always relative to the absolute 
magnetic north pole of the earth. However, the compass data is sensitive to magnetic anomaly caused by the magnetic field emanating from the operating thruster motors and the man-made underwater structures, which contains reinforcing iron rods. Figure 19 shows the heading angles measured by gyro and compass while the mosaicing experiments. The magnetic anomaly causes the fluctuation of measured heading angles that cause the rotation of the source images. Therefore the image stitching algorithm must rotate the images in opposite directions before stitching them in this situation. In contrast, the heading angles measured by gyro are relatively stable, and this characteristic minimizes the rotation of the source images. Although gyro has drift error, the amount of drift is not significantly high for short-term operation as mentioned in Section 2.4.

A landmark to be recognized by a vision tracking system was attached on the top of Cyclops. The trajectories of Cyclops were measured using this landmark tracking, and the results were shown in Fig. 20. Even though the trajectory with the constant heading navigation did not exactly coincide with the reference trajectory, the error accumulation was low compared to that of the variable heading navigation. For a quantitative comparison, the distance and angle deviations of both cases are graphed in Fig. 21. The distance and angle deviations in the variable heading navigation continuously increased when passing through the waypoints. In contrast, the distance and angle deviations in the constant heading navigation was relatively low. Therefore, the proposed strategy was effective to reduce the dead reckoning error during image mosaicing.

A total of 81 source images were obtained in these experiments, and we 
produced a mosaic image using Microsoft Image Composite Editor, which is freeware designed for image mosaicing. The results are shown in Fig. 22. The actual resolution of the image is $10,434 \times 10,471$ pixels, and it shows the entire area of the printed cotton sheet.

Figure 23 shows enlarged images of the mosaic image in Fig. 22. In these images, the grid patterns and characters are well-connected, without large pixel misalignment.

To analyze the details of mosaiced images, the source images in Fig. 22 (a) and (b) were manually arranged as shown in Figs. 24 and 25, respectively. The areas of overlap are represented as bright regions. According to the proposed mosaicing scenarios, the areas of overlap have to be homogeneously distributed in the images. From Fig. 24 and 25, the constant heading navigation provides homogeneous distribution of the areas of overlap at a glance. For quantitative comparison, the waypoints of each image were separated to eight groups and the waypoints in each group were combined and formed as line by linear interpolation. The $\mathrm{R}$-square value of each line are shown in Table 4. Then, the distance and angle between adjacent lines were calculated and graphed in Figs. 26 (a) and (b), respectively. The distance between adjacent lines in the constant heading navigation were almost the same, but the distance between adjacent lines in the variable heading navigation were fluctuated. The heading angle deviations of the variable heading navigation were higher than those of the constant heading navigation. As a result, the constant heading navigation is practically more effective to collect homogeneous source images for image mosaicing. 


\subsection{Field test and Future Work}

A field test was conducted in Yeongil Bay, Pohang, South Korea, as shown in Fig. 27. Similar to the image mosaicing experiment conducted in the indoor water tank, Cyclops was controlled to maintain a constant heading during image mosaicing. Moreover, Cyclops was planned to follow the lawnmower trajectories at a constant altitude of $2 m$ above the seafloor. Figure 28 shows the lawnmower trajectory of Cyclops while the field test. Cyclops covered the $15 m \times 5 m$ seafloor area.

The heading angle deviation during this experiment is graphed in Fig. 29. For one hour, the heading angle deviation became $9.7^{\circ}$ which is relatively higher than that of the indoor test. The increase of the heading angle deviation was caused by disturbances such as underwater currents. To overcome the deviation, advanced control and navigation algorithms or external infrastructures, such as USBL, are required.

Figure 30 shows the result of image mosaicing whose actual resolution is $24,912 \times 8,901$ pixels. The number of source images stitched for the mosaicing is 253. Through the mosaicing image, we can see some man-made fish banks and sea urchins whose sizes are about $2 \mathrm{~m}$ and $7 \mathrm{~cm}$, respectively.

Cyclops has a high potential for underwater sensing. Unlike the conventional sensing methods, which measure point-wise data using submerged sensors, Cyclops can measure spatially distributed data, which is in a more readily usable form for humans. For example, the location of a chemical pollution source can be suggested based on the gradient of measurements obtained using various sensors such as temperature, salinity, and turbidity sensors, which will be mounted on Cyclops. Then, the underwater measure- 
ments will be obtained by Cyclops based on the proposed strategy.

\section{Conclusion}

In this paper, we discussed the development of a hovering-type AUV called Cyclops. The body of Cyclops has a symmetrical structure, and it uses a propulsion system for motions in the heave, surge, and sway directions to achieve the proposed strategy. To verify its sensing performance, we executed underwater image mosaicing in both an indoor water tank and the field. For image mosaicing, the path of Cyclops was planned, and it was controlled to track the path without varying its heading direction. As a result, we obtained source images with sufficient areas of overlap, no perspective distortion, and homogeneous optical conditions. Therefore, panoramic images could be readily generated using general image mosaicing software.

\section{Acknowledgements}

This research was supported by the MSIP(Ministry of Science, ICT and Future Planning), Korea, under the "IT Consilience Creative Program" (NIPA2014-H0201-14-1001), supervised by the NIPA(National IT Industry Promotion Agency). This work was also partly supported by the Civil Military Technology Cooperation Center.

\section{Reference}

Advantech, 2014. www. advantech.com, accessed: 2014-02-20. 
Alippi, C., Camplani, R., Calperti, C., Roveri, M., January 2011. A robust, adaptive, solar-powered WSN framework for aquatic environmental monitoring. IEEE Sensors Journal 11 (1), 45-55.

Bian, X., Yan, Z., Chen, T., Yu, D. L., Zhao, Y., 2012. Mission management and control of BSA-AUV for ocean survey. Ocean Engineering 55, 161-174.

Caccia, M., 2007. Vision-based ROV horizontal motion control: Near-seafloor experimental results. Control engineering practice 15 (6), 703-714.

Caccia, M., Veruggio, G., 2000. Guidance and control of a reconfigurable unmanned underwater vehicle. Control Engineering Practice 8 (1), 21-37.

Diamond systems, 2014. www.diamondsystems.com, accessed: 2014-02-20.

Faugeras, O., Luong, O. T., 2001. The Geometry of Multiple Images: The Laws That Govern the Formation of Multiple Images of a Scene and Some of Their Applications. Cambridge, MA: MIT Press.

Fleischer, S. D., Rock, S. M., Burton, R. L., 1997. Global position determination and vehicle path estimation from a vision sensor for real-time video mosaicking and navigation. In: Proceedings of the MTS/IEEE OCEANS 97 Conference.

Fossen, T. I., 1994. Guidance and control of ocean vehicles. New York: Wiley.

Griffiths, G., 2003. Technology and Applications of Autonomous Underwater Vehicles. New York, Taylor \& Trance Inc.

Hayato, K., Ura, T., 2004. Navigation of an AUV for investigation of underwater structures. Control engineering practice 12 (12), 1551-1559. 
Hayato, K., Yu, S., Ura, T., 2001. Object observation in detail by the auv "Tri-Dog 1" with laser pointers. In: Proc. of OCEANS, 2001. MTS/IEEE Conference and Exhibition. pp. 390-396.

Honeywell, 2014. www . honeywell. com, accessed: 2014-02-20.

Hyakudome, T., Aoki, T., Murashima, T., Tsukioka, S., Yoshida, H., Nakajoh, H., Ida, T., Ishibashi, S., Sasamoto, R., 2002. Key technologies for AUV "URASHIMA". In: Proc. of MTS/IEEE Oceans. pp. 162-166.

Imagenex, 2014. www . imagenex.com, accessed: 2014-02-20.

Jaffe, J. S., 1990. Computer modeling and the design of optimal underwater imaging system. IEEE Journal of Oceanic Engineering 15 (2), 101-111.

Kim, M., Joe, H., Kim, J., Kim, H., Lee, J. S., Yu, S.-C., 2013. Variablestructure PID controller with anti-windup for autonomous underwater vehicle. In: Proc. MTS/IEEE OCEANS Conf.

Kim, T. W., Yuh, J., 2004. Development of a real-time control architecture for a semi-autonomous underwater vehicle for intervention missions. Control engineering practice 12 (12), 1521-1530.

Kojima, J., 2003. Cable tracking by autonomous underwater vehicle. In: Proc. of The 3rd International Workshop on Scientific Use of Submarine Cables and Related Technologies. pp. 171-174.

Kongberg, 2014. www .km .kongsberg. com/cameras, accessed: 2014-02-20. 
Kumagai, M., Ura, T., Kuroda, Y., Walker, R., October 2002. A new autonomous underwater vehicle designed for lake environment monitoring. Advanced Robotics 16 (1), 17-26.

Li, J.-H., Park, S.-K., Oh, S.-S., Suh, J.-H., Yoon, G.-H., Baek, M.-S., 2011. Development of a Hovering-Type Intelligent Autonomous Underwater Vehicle, P-SURO. In the Book Autonomous Underwater Vehicles, InTech Education and Publishing, pp. 21-38.

Ludvigsen, M., Sortland, B., Johnsen, G., Singh, H., 2007. Applications of geo-referenced underwater photo mosaics in marine biology and archaeology. IEEE Journal of Oceanic Engineering 20 (4), 140-149.

Marani, G., Choi, S. K., Yuh, J., 2009. Underwater autonomous manipulation for intervention missions AUVs. Ocean Engineering 36 (1), 15-23.

Marks, R. L., Lee, M. J., Rock, S. M., 1994a. Using visual sensing for control of an underwater robotic vehicle. In: Proceedings of IARP Second Workshop on Mobile Robots for Subsea Environments.

Marks, R. L., Rock, S. M., Lee, M. J., 1994b. Real-time video mosaicking of the ocean floor. In: Proceedings of IEEE Symposium on Autonomous Underwater Vehicle Technology.

Marks, R. L., Rock, S. M., Lee, M. J., July 1995. Real-time video mosaicking of the ocean floor. IEEE Journal of Oceanic Engineering 20 (3), 229-241.

Mensor, 2014. www.mensor.com, accessed: 2014-02-20.

Misumi catalog, 2013. us.misumi-ec.com, accessed: 2014-02-20. 
Nasahashi, K., Ura, T., Asada, A., Obara, T., Sakamaki, T., Kim, K., Okamura, K., 2005. Underwater volcano observation by autonomous underwater vehicle "R2D4". In: Proc. of OES OCEANS. pp. 557-562.

Novatel, 2014. www .novatel.com, accessed: 2014-02-20.

Pizarro, O., Singh, H., October 2003. Toward large-area underwater mosaicing for scientific applications. IEEE Journal of Oceanic Engineering 28 (4), 651-672.

Prados, R., Garcia, R., Gracias, N., Escartin, J., Neumann, L., October 2012. A novel blending technique for underwater gigamosaicing. IEEE Journal of Oceanic Engineering 37 (4).

Pyo, J., 2012. Development of the hovering type AUV 'Cyclops' for underwater sensor fusion. Master's thesis, Pohang University of Science and Technology, written in Korean.

Robert, E., Sanders, J., 2001. Technology innovation in aluminum products. The Member Journal of TMS 53 (2), 21-25.

Singh, H., Howland, J., Pizarro, O., July 2004. Advances in large-area photo mosaicking underwater. IEEE Journal of Oceanic Engineering 29 (3).

Sisman, D., 1982. The professional diver's handbook. submex, U.K.

Sound Metrics, 2014. www. soundmetrics.com, accessed: 2014-02-20.

Szeliski, R., 1994. Image Mosaicing for Tele-Reality Applications. Cambridge Research Laboratory, Cambridge, MA, Tech. Rep. CRL 94/2. 
Tecnadyne, 2014. www.tecnadyne.com, accessed: 2014-02-20.

Teledyne, 2014. teledyne.com, accessed: 2014-02-20.

Thornton, B., Ura, T., Nose, Y., Turnock, S., 2007. Zero-G class underwater robots: Unrestricted attitude control using control moment gyros. IEEE Journal of Oceanic Engineering 32 (3), 565-583.

Ura, T., Kumagai, M., Sakakibara, T., Kimura, Y., Okumura, T., Kazuyoshi, S., Sasaki, M., Matsushima, M., 2002. Construction and operation of four autonomous underwater vehicles for lake survey. In: Proc. of the International Symposium on Underwater Technology. pp. 24-29.

Ura, T., Kurimoto, Y., Kondo, H., Nose, Y., Sakamaki, T., Kuroda, Y., 2005. Observation behavior of an AUV for ship wreck investigation. In: Proc. of MTS/IEEE OCEANS. pp. 2686-2691.

Vaganay, J., Elkins, M., Esposito, D., O'alloran, W., Hover, F., Kokko, M., 2006. Ship hull inspection with the HAUV: US navy and NATO demonstrations results. In: Proc. of OCEANS MTS/IEEE Conference and Exhibition. pp. 1-6.

Whitcomb, L. L., April 2009. Underwater robotics: Out of the research laboratory and into the field. In: Proc. of the International Conference on Robotics and Automation. pp. 709-716.

Yu, S. C., Kim, T. W., Marani, G., Choi, S. K., April 2007. Real-time 3D sonar image recognition for underwater vehicles. In: Symposium on Underwater Technology and Workshop on Scientific Use of Submarine Cables and Related Technologies. pp. 142-146. 
Yu, S. C., Teng, Z., Kang, D. J., September 2012. Modeling of high-resolution 3D sonar for image recognition. Int. J. of Offshore and Polar Eng. 22 (3), $1-7$.

Yuh, J., 1995. Underwater Robotic Vehicles: Design and Control. New Mexico: TSI Press.

Yuh, J., 2000. Design and control of autonomous underwater robots: A survey. Autonomous Robot 8 (1), 7-24. 


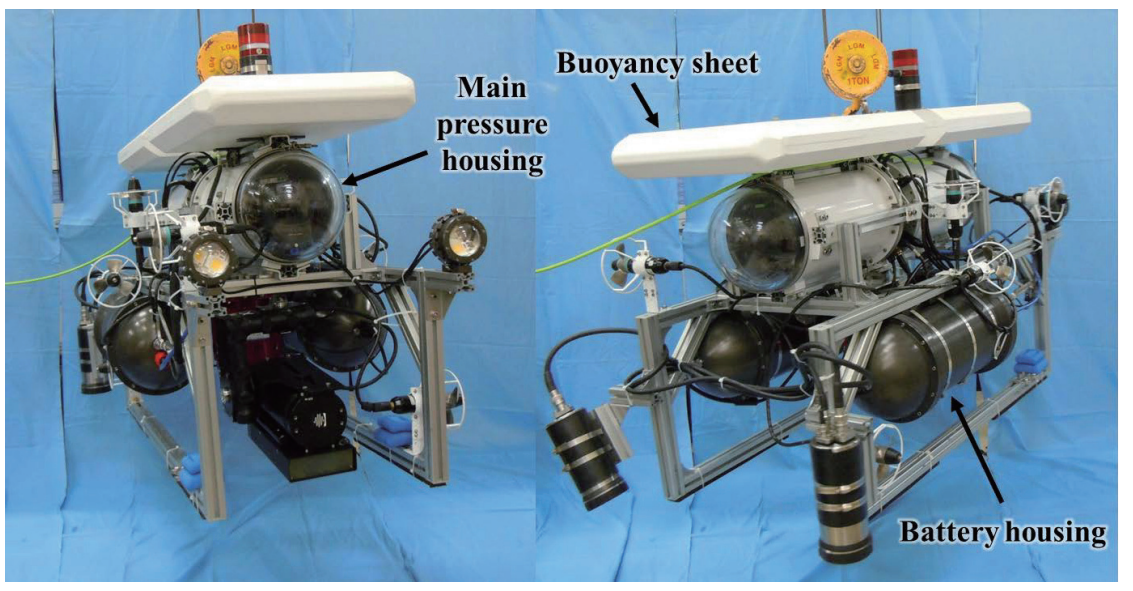

Figure 1: Hovering-type AUV: Cyclops. 
Table 1: Specifications of Cyclops.

\begin{tabular}{ll}
\hline Dimension & $900 \mathrm{~mm} \times 1500 \mathrm{~mm} \times 900 \mathrm{~mm}$ \\
\hline Weight & $210 \mathrm{~kg}$ in air \\
\hline Depth Rating & $100 \mathrm{~m}$ \\
\hline Propulsion & 8 thrusters $(475 \mathrm{~W})$ \\
\hline Maximum Speed & 2 knots \\
\hline Power Source/Batteries & $24 \mathrm{VDC} / 600 \mathrm{Wh}$ Li-Po battery $\times 2$ \\
\hline Computer System & PC-104 $($ Intel Atom working at $1.66 \mathrm{GHz}) \times 2$ \\
\hline & $1.1 \mathrm{MHz} / 1.8 \mathrm{MHz}$ imaging sonar \\
& Profiling sonar $(675 \mathrm{kHz}-1 \mathrm{MHz})$ \\
& Digital pressure transducer \\
& Doppler velocity log $(1.2 \mathrm{MHz})$ \\
& High resolution still camera $(10 \mathrm{Mpixels})$ \\
& Strobe light \\
& Fiber-optic gyro \\
& Pan-tilt camera $\times 2$ \\
\hline
\end{tabular}




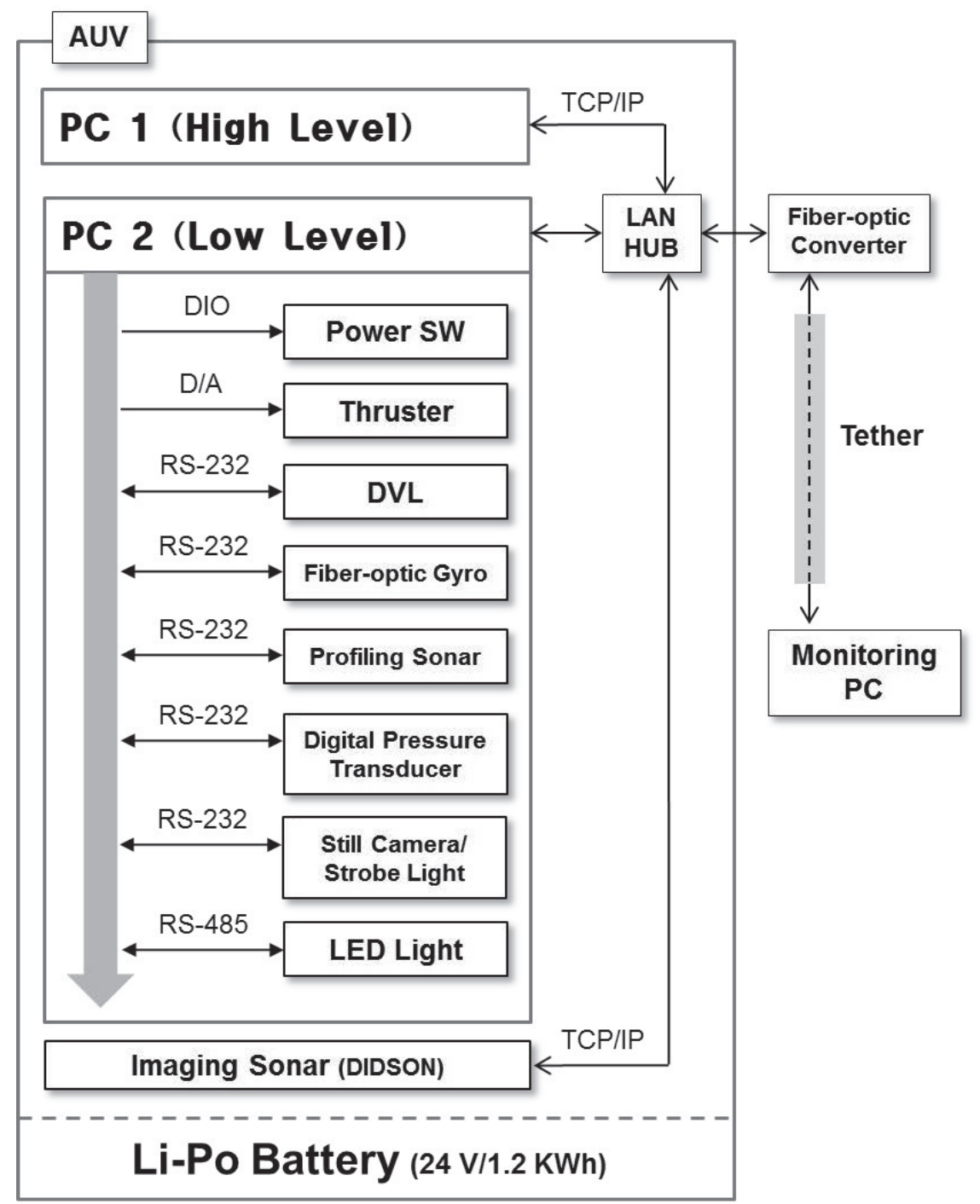

Figure 2: Configuration of communication interfaces and power lines between components. 


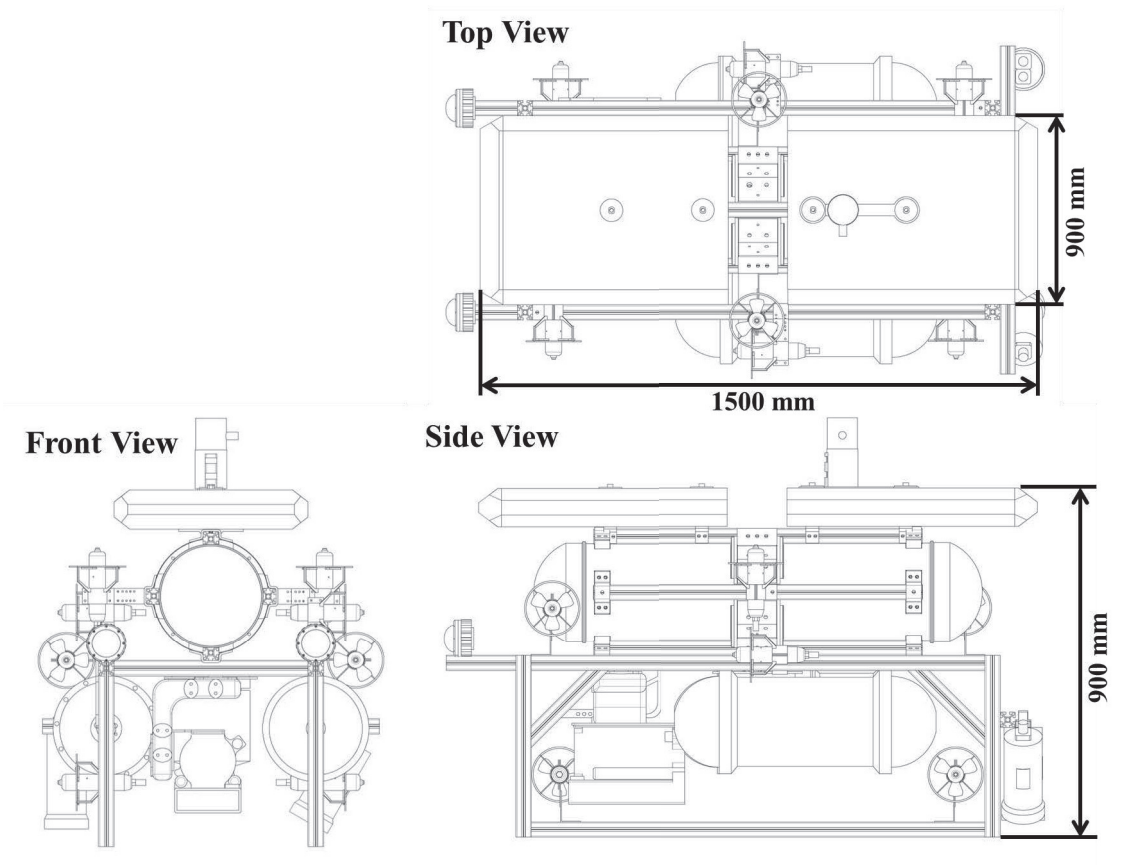

Figure 3: Layout of Cyclops. 


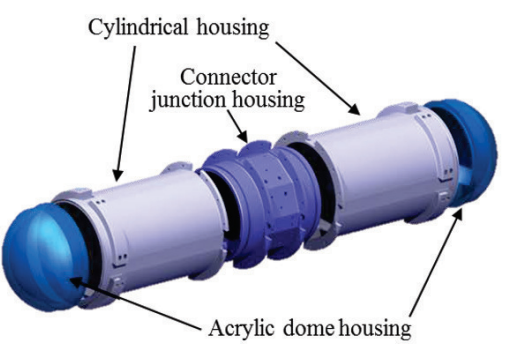

(a) Main pressure housing

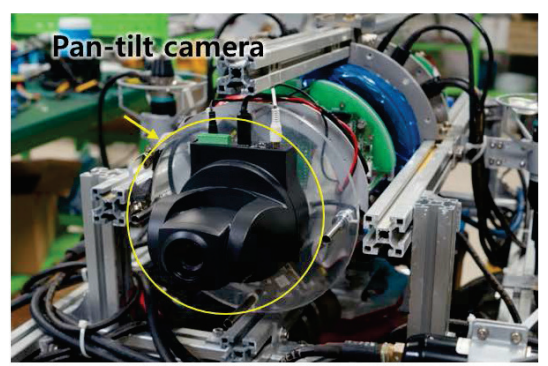

(c) Pan-tilt camera

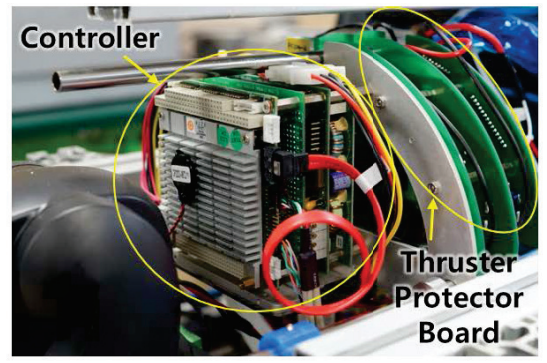

(b) Controller

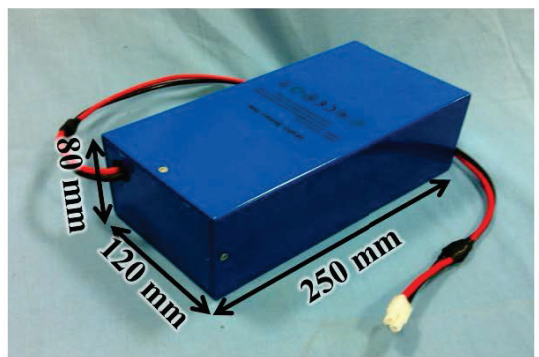

(d) Battery pack

Figure 4: Principal hardware components of Cyclops. 


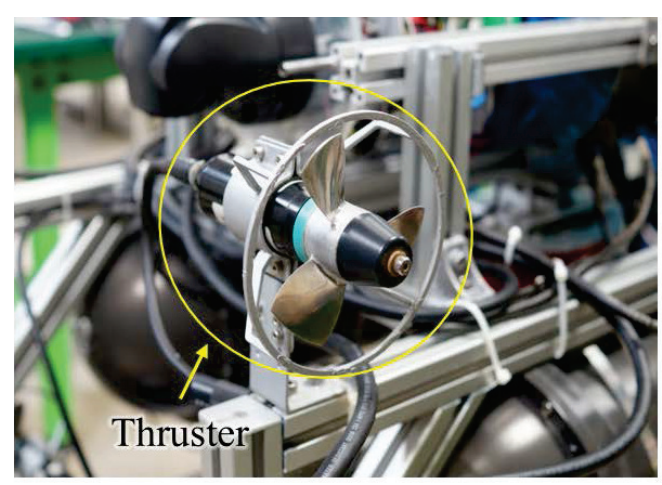

(a) Thruster

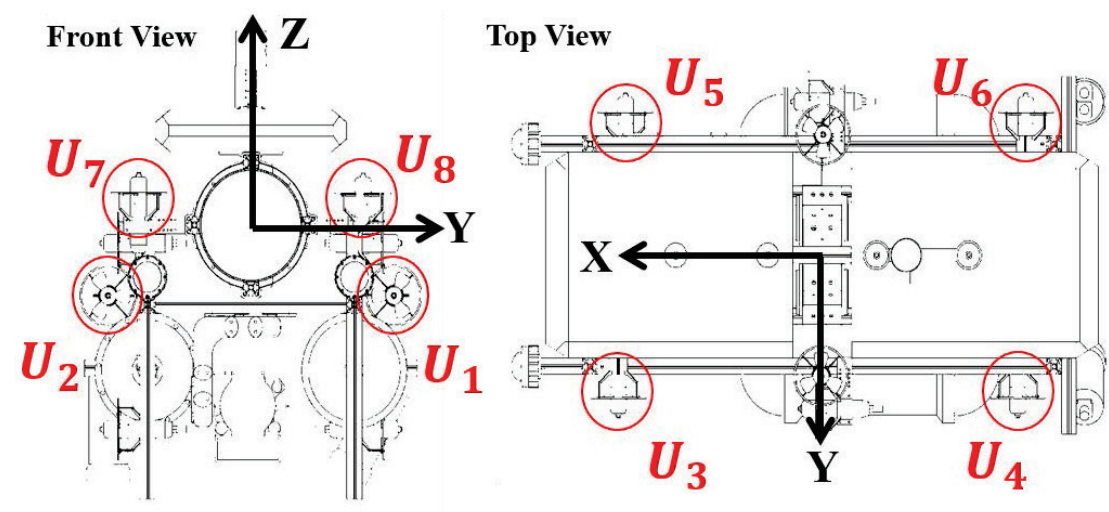

(b) Configuration of thrusters

Figure 5: Thrusters used for Cyclops and their configuration. 

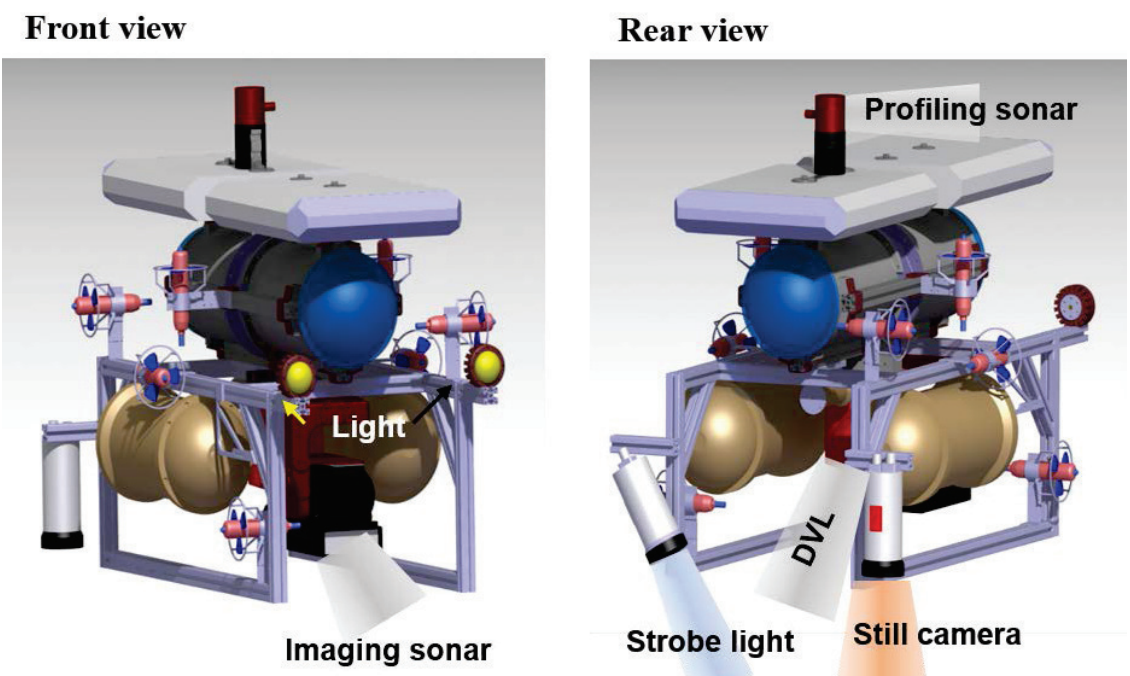

Figure 6: Configuration of sensors. 


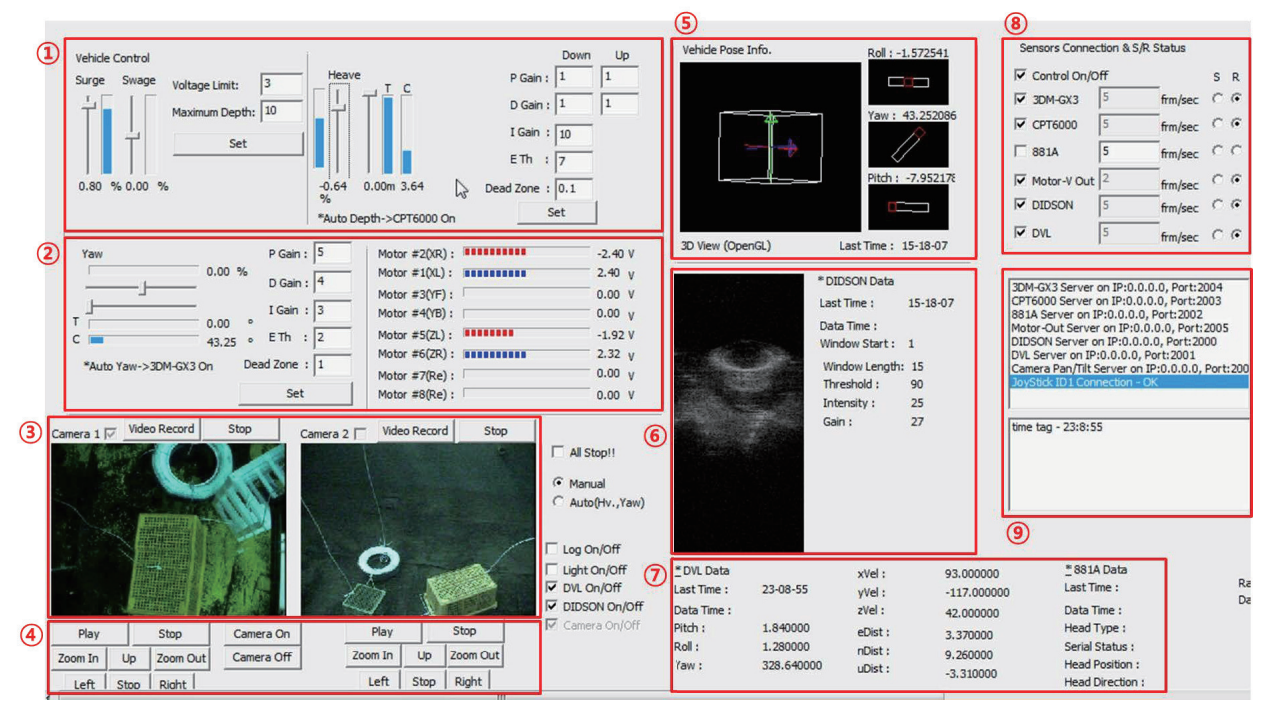

Figure 7: Graphic user interface of software for operating Cyclops. (1) Manual controller: The automatic control mode is changed to the manual mode. (2) Status of propulsion system: The output power of each thruster is monitored in real time. (3) Optical video images: The video images from the front and real cameras are displayed in real time. (4) Camera controller: The pan and tilt angles of the front and rear cameras are adjusted. (5) Attitude of Cyclops: The current pan, tilt, and yaw angles of Cyclops are monitored. (6) Acoustic image: The sonar image is displayed in real time. (7) System status: The current depth, velocity, and position of Cyclops are monitored. (8) Sensor controller: The sensors of Cyclops can be enabled and disabled. (9) Communication status: The status of the communication between the sensors and controllers is displayed. 


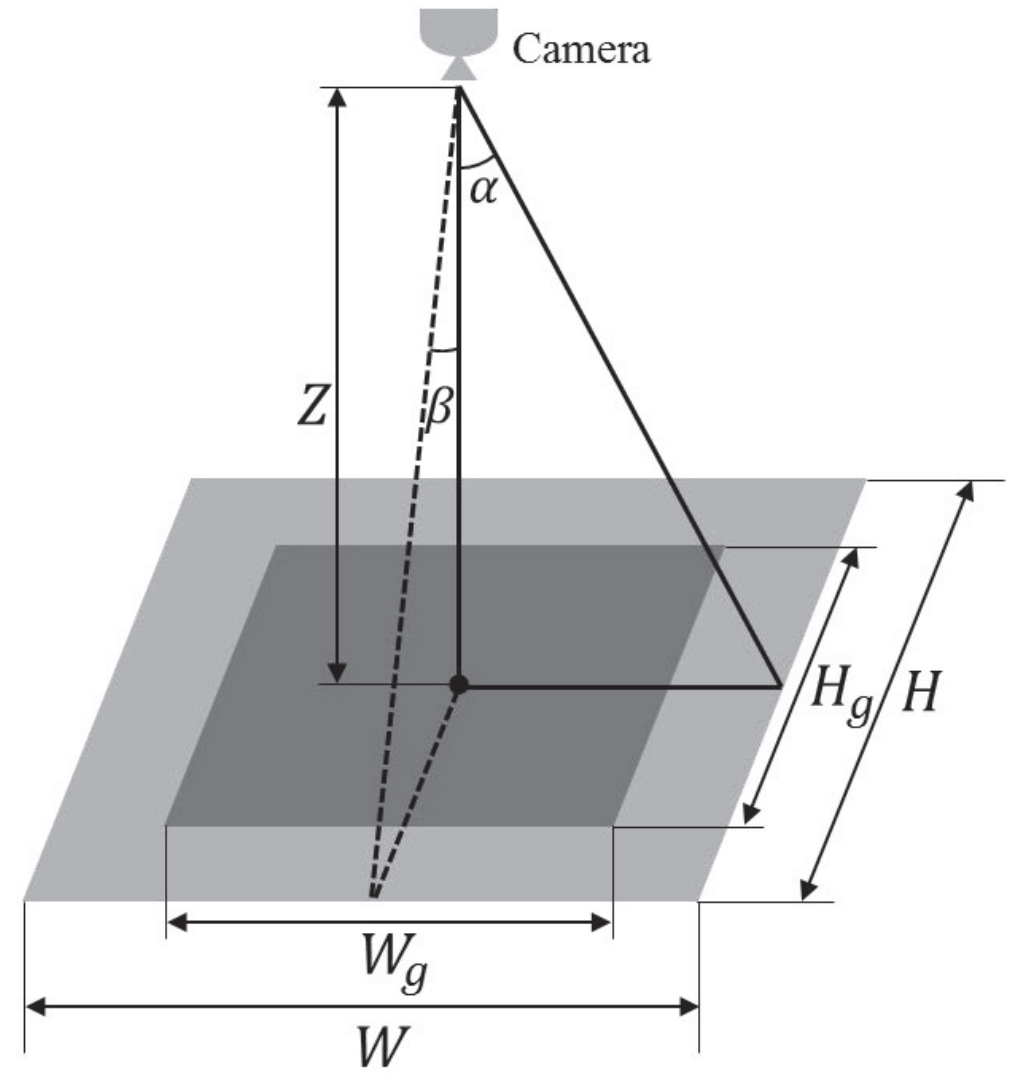

Figure 8: Camera's field of view. 


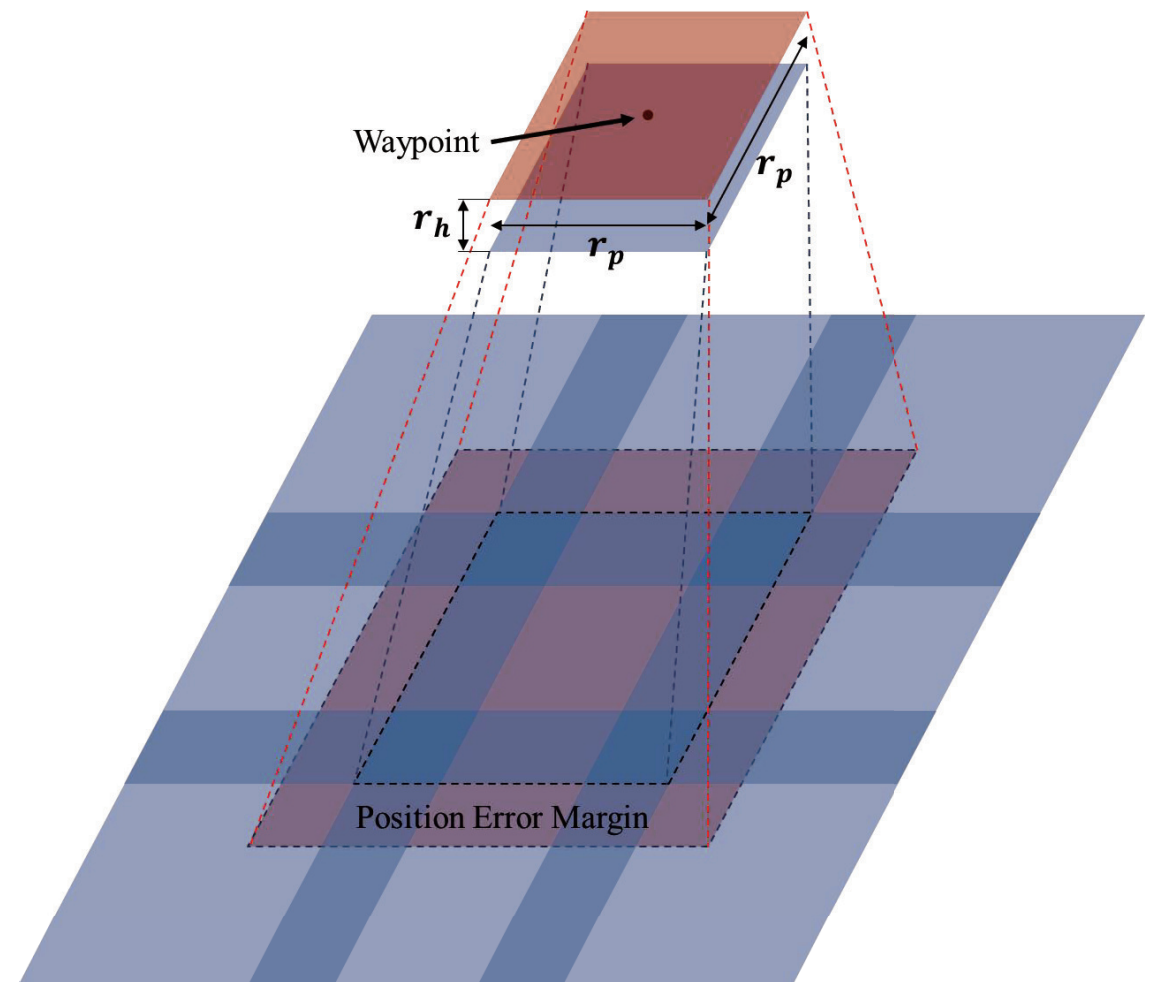

Figure 9: Position error margin defined as cuboid area. 


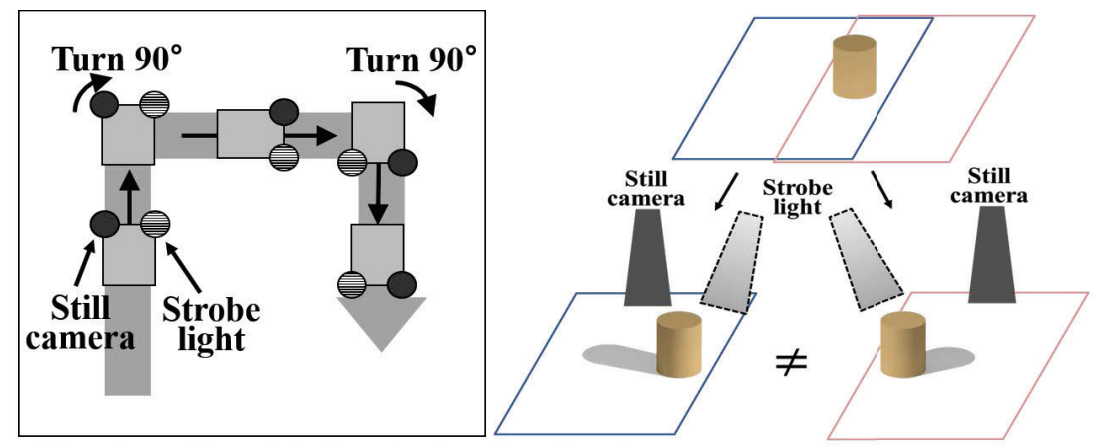

(a) Path tracking with changing heading direction

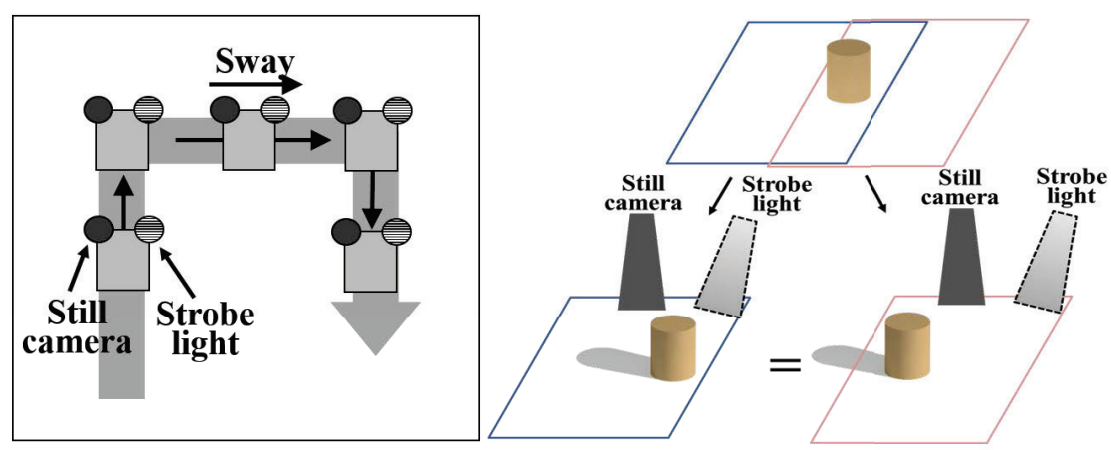

(b) Navigation without changing heading direction

Figure 10: Navigation methods with and without changing heading direction. 


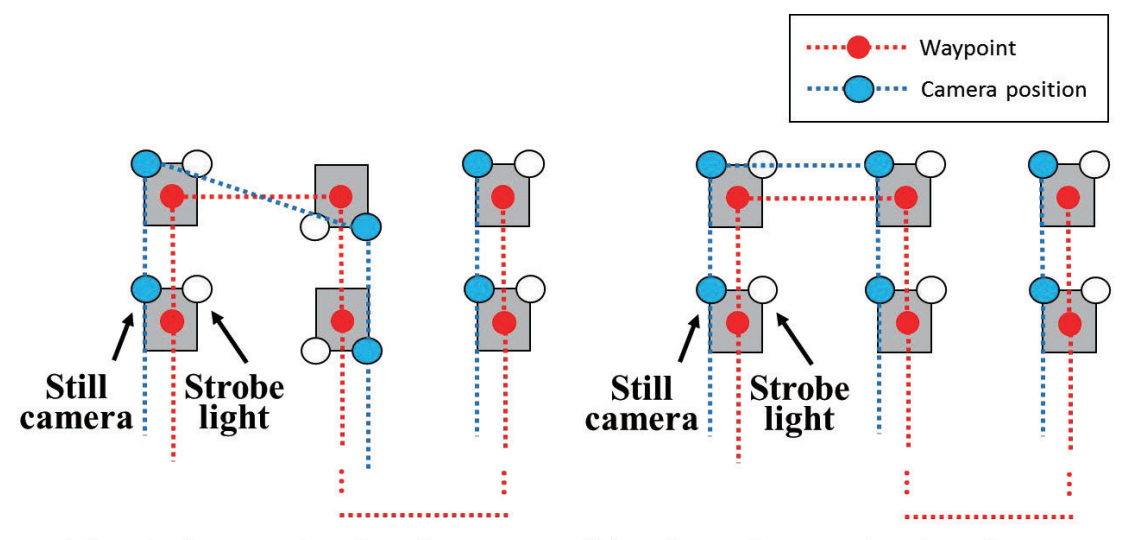

$\begin{array}{ll}\text { (a) with changing heading direction } & \text { (b) without changing heading direction }\end{array}$

Figure 11: Relative position between camera and waypoint during navigation. 


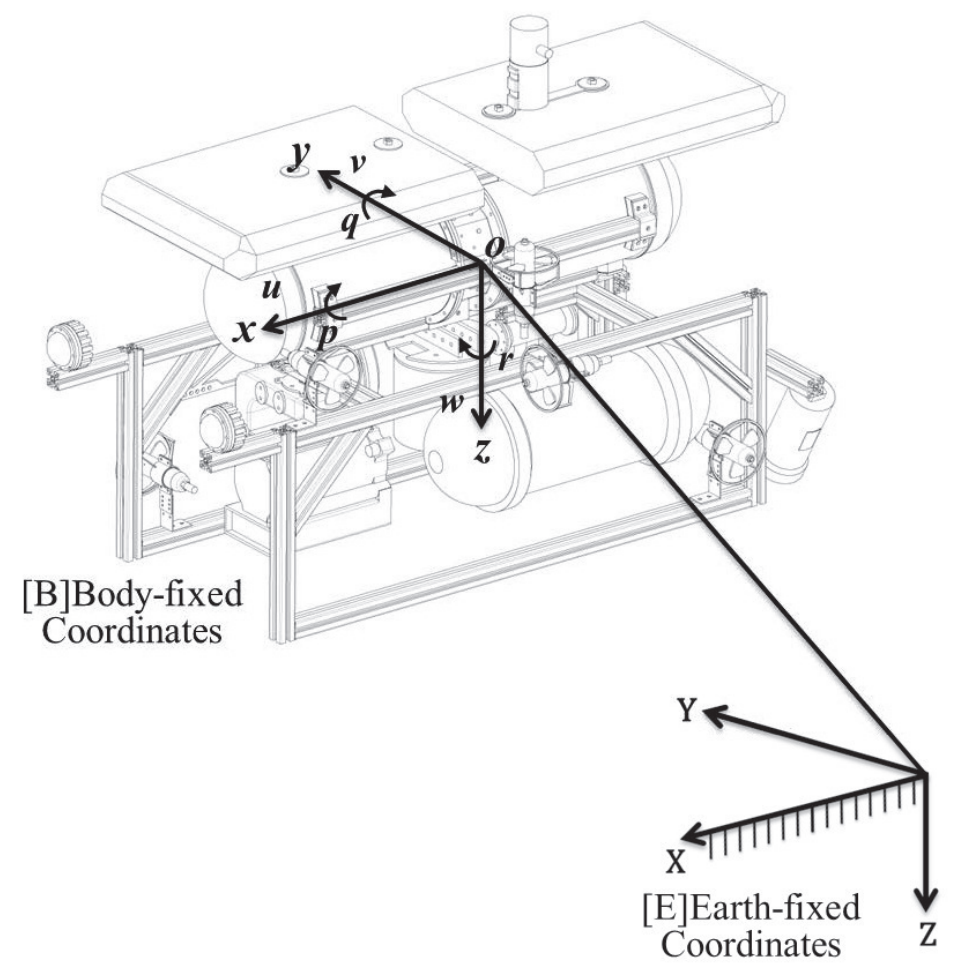

Figure 12: Coordinates system. 


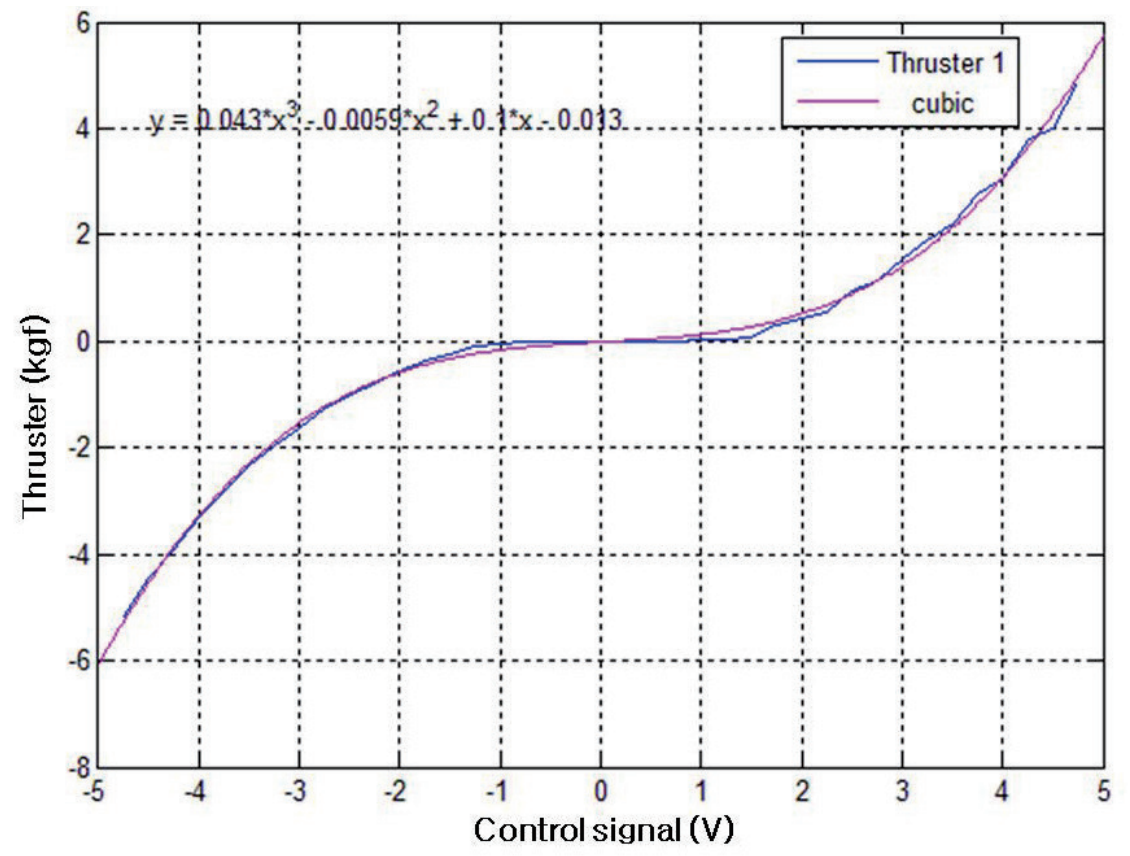

Figure 13: Calibration result for thruster 1. 
Table 2: Parameters of each thruster.

\begin{tabular}{lcccccc}
\hline & $c_{1 i-}$ & $c_{2 i-}$ & $c_{3 i-}$ & $c_{1 i+}$ & $c_{2 i+}$ & $c_{3 i+}$ \\
\hline$u_{1}$ & -0.3266 & -0.5388 & -0.2919 & 0.3027 & -0.4609 & 0.1516 \\
$u_{2}$ & 0.2867 & 0.3047 & 0.033 & -0.2978 & 0.4097 & -0.1413 \\
$u_{3}$ & 0.2889 & 0.3873 & 0.086 & -0.3256 & 0.5468 & -0.2179 \\
$u_{4}$ & 0.2744 & 0.3703 & 0.099 & -0.3524 & 0.6428 & -0.3325 \\
$u_{5}$ & -0.2986 & -0.3567 & -0.075 & 0.3168 & -0.4875 & 0.1412 \\
$u_{6}$ & -0.2673 & -0.3137 & -0.073 & 0.3304 & -0.5153 & 0.1859 \\
$u_{7}$ & -0.2673 & -0.3137 & -0.073 & 0.3304 & -0.5153 & 0.1859 \\
$u_{8}$ & -0.2673 & -0.3137 & -0.073 & 0.3304 & -0.5153 & 0.1859 \\
\hline
\end{tabular}




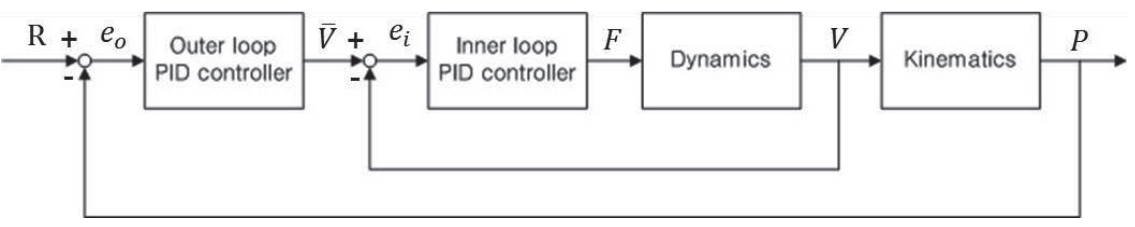

Figure 14: Block diagram of proposed controller. 


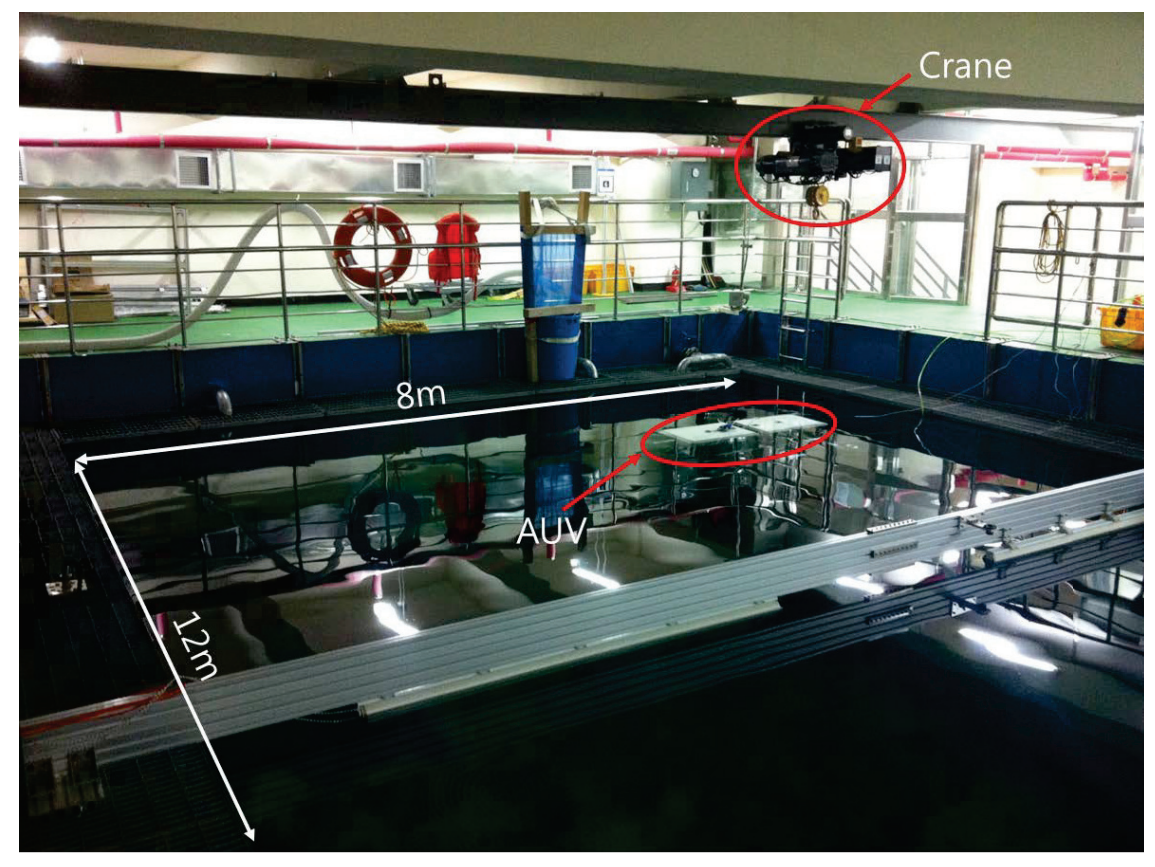

Figure 15: Indoor water tank for control system test. 
Table 3: PID gains for surge, sway, heave and yaw motions.

\begin{tabular}{ccccc}
\hline & Surge & Sway & Heave & Yaw \\
\hline$K_{P o}$ & 2.5 & 2.5 & 2.2 & 0.8 \\
$K_{D o}$ & 0 & 0 & 0.001 & 0.001 \\
$K_{I o}$ & 2 & 0 & 0 & 0.1 \\
$K_{P i}$ & 0.3 & 0.3 & 2 & 1 \\
$K_{D i}$ & 0 & 0 & 0 & 0 \\
$K_{I i}$ & 0 & 0 & 0 & 0 \\
\hline
\end{tabular}




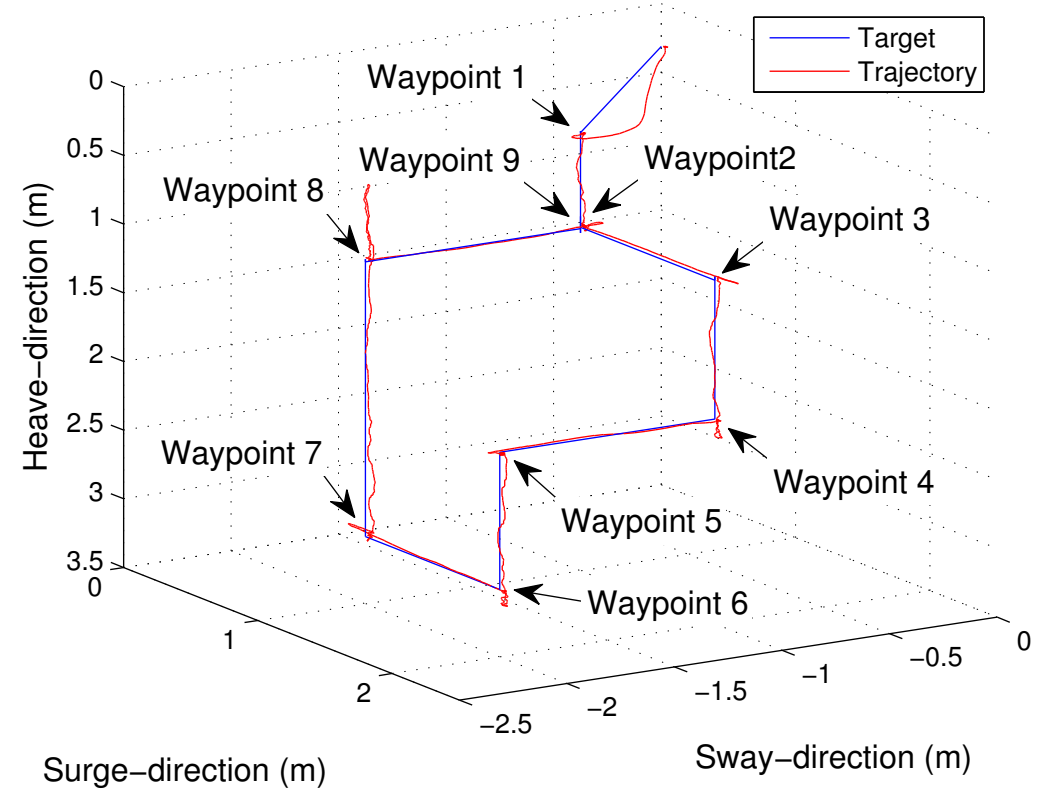

Figure 16: Path and waypoint definition and path-tracking experiment results. 

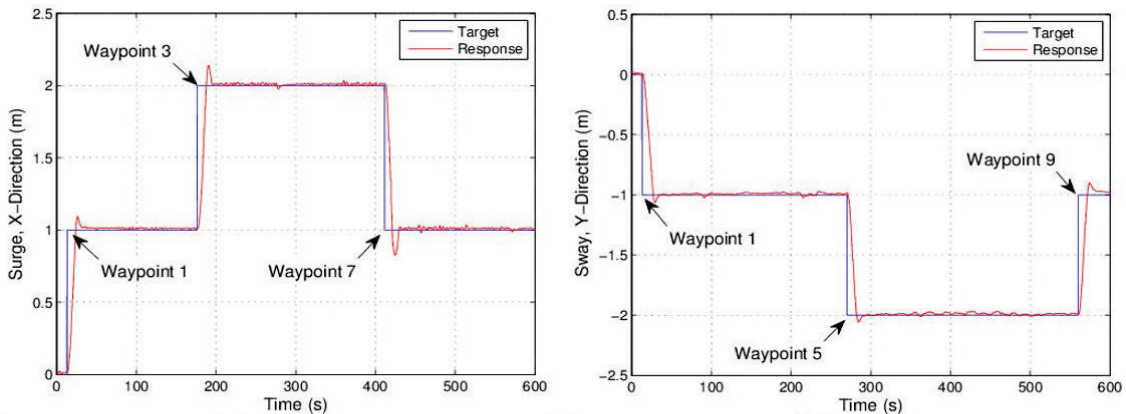

(a) Surge

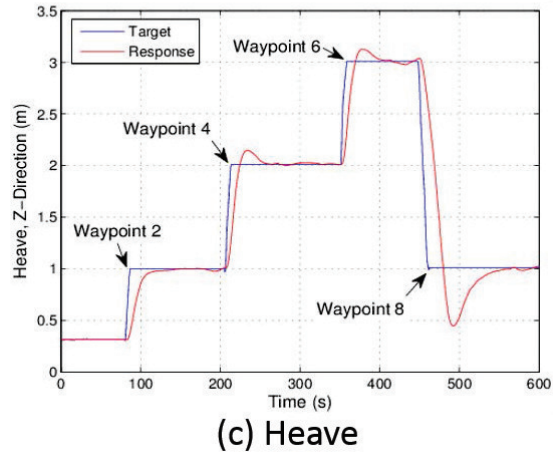

(b) Sway

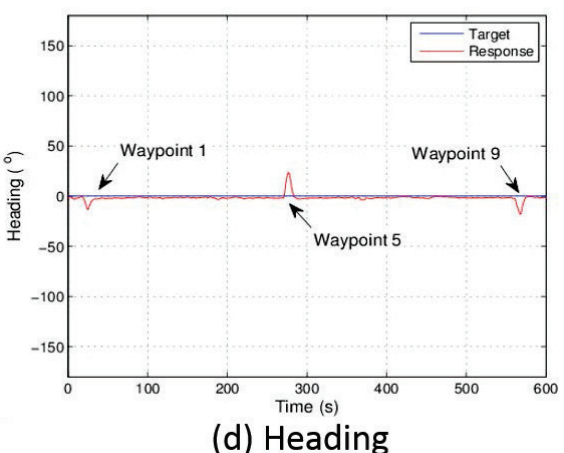

Figure 17: Comparisons between desired path and trajectory of Cyclops in surge, sway, heave, and yaw directions. 


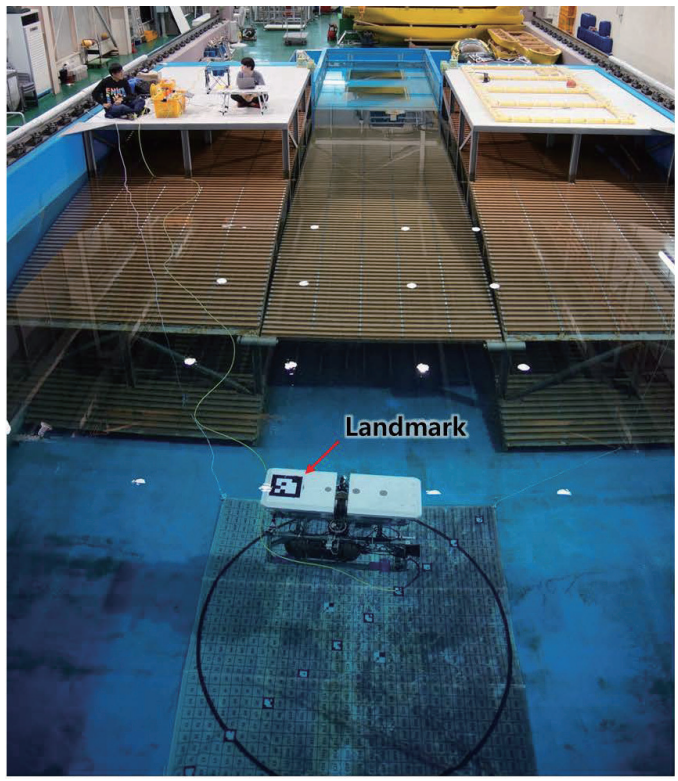

(a) Image mosaicing experiment environment

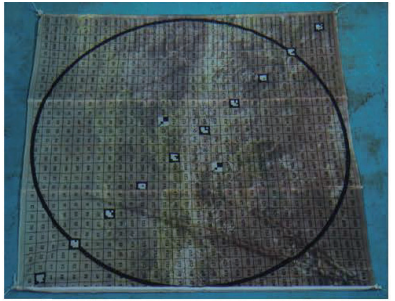

(b) Designed and printed flag

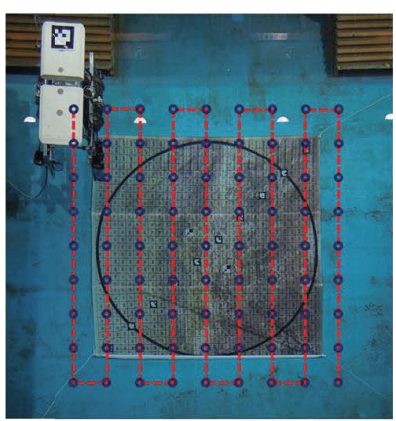

(c) Lawnmower trajectory

Figure 18: Experiment setup for image mosaicing. 


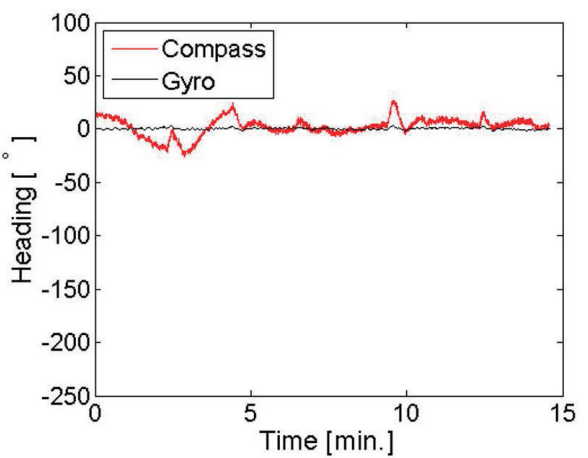

(a) Constant heading navigation

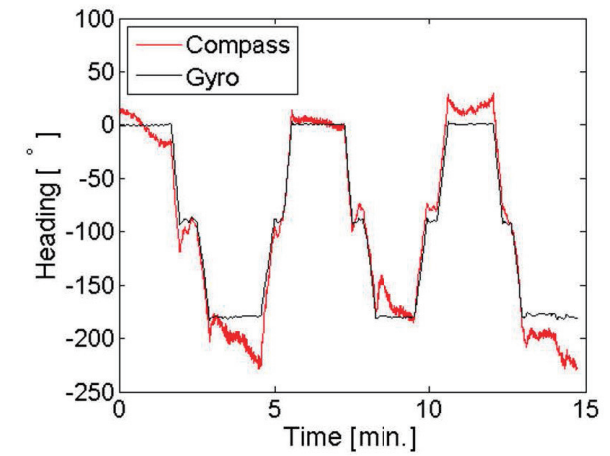

(b) Variable heading navigation

Figure 19: Heading angles measured by gyro and compass while mosaicing experiments. 

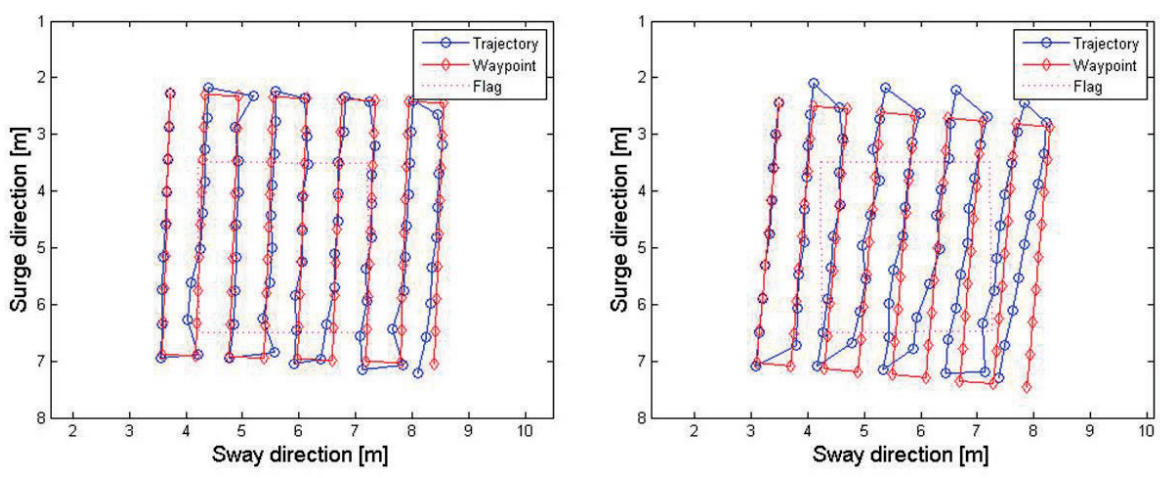

(a) Trajectory of constant heading

(b) Trajectory of variable heading

Figure 20: Trajectories of mosaicing experiments. 


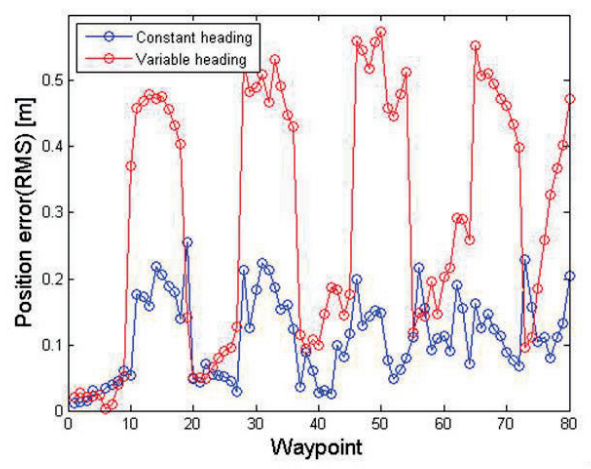

(a) Position deviation

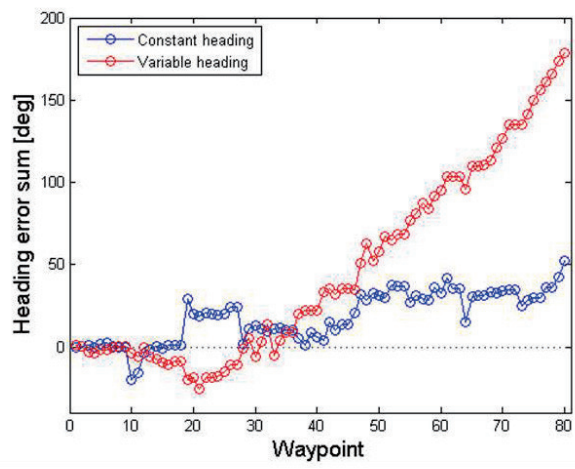

(b) Heading deviation

Figure 21: Position and heading angle deviations of trajectories. 


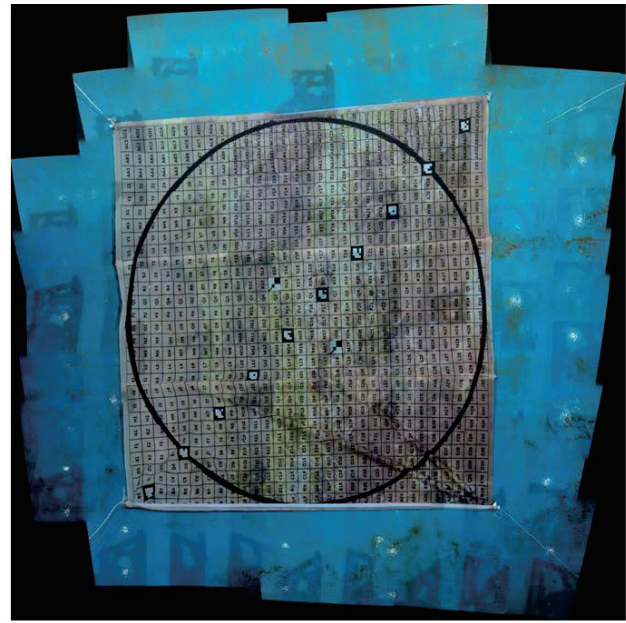

(a) Image mosaicing result of constant heading

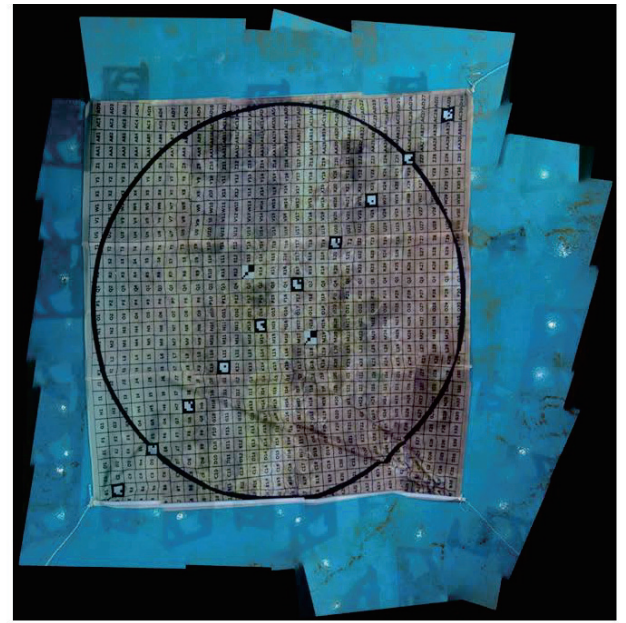

(b) Image mosaicing result of variable heading

Figure 22: Image mosaicing results. 


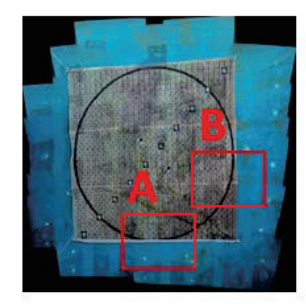

Constant heading

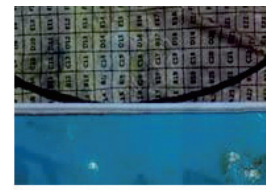

(a) Position A

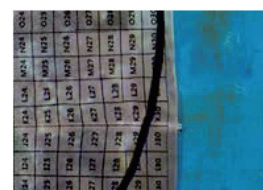

(b) Position B

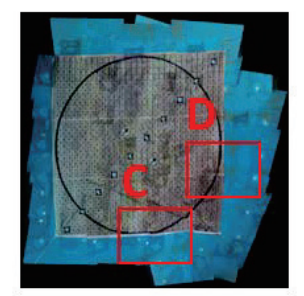

Variable heading

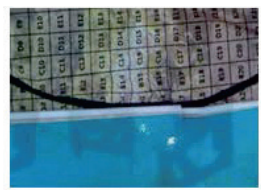

(c) Position C

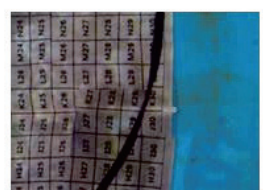

(d) Position D

Figure 23: Enlarged images for checking connectivity accuracy. 
Table 4: R-square value of each linear interpolation.

\begin{tabular}{ccccccccc}
\hline Waypoint & 1 & 2 & 3 & 4 & 5 & 6 & 7 & 8 \\
\hline Constant Heading & 0.917 & 0.912 & 0.865 & 0.874 & 0.973 & 0.948 & 0.989 & 0.984 \\
Varying Heading & 0.685 & 0.939 & 0.738 & 0.960 & 0.890 & 0.980 & 0.990 & 0.991 \\
\hline
\end{tabular}




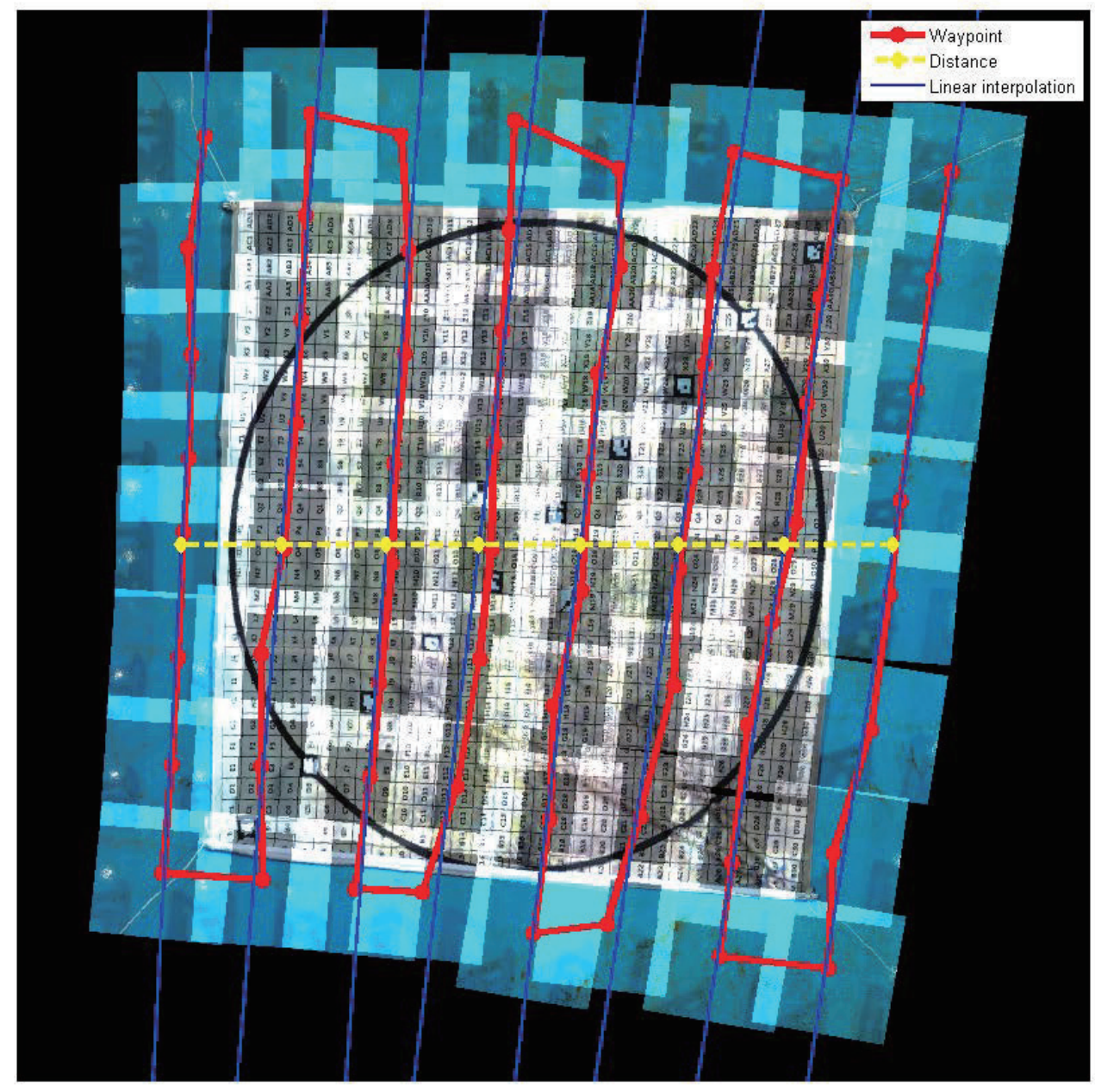

Figure 24: Source image arrangement in constant heading angle during scanning. 


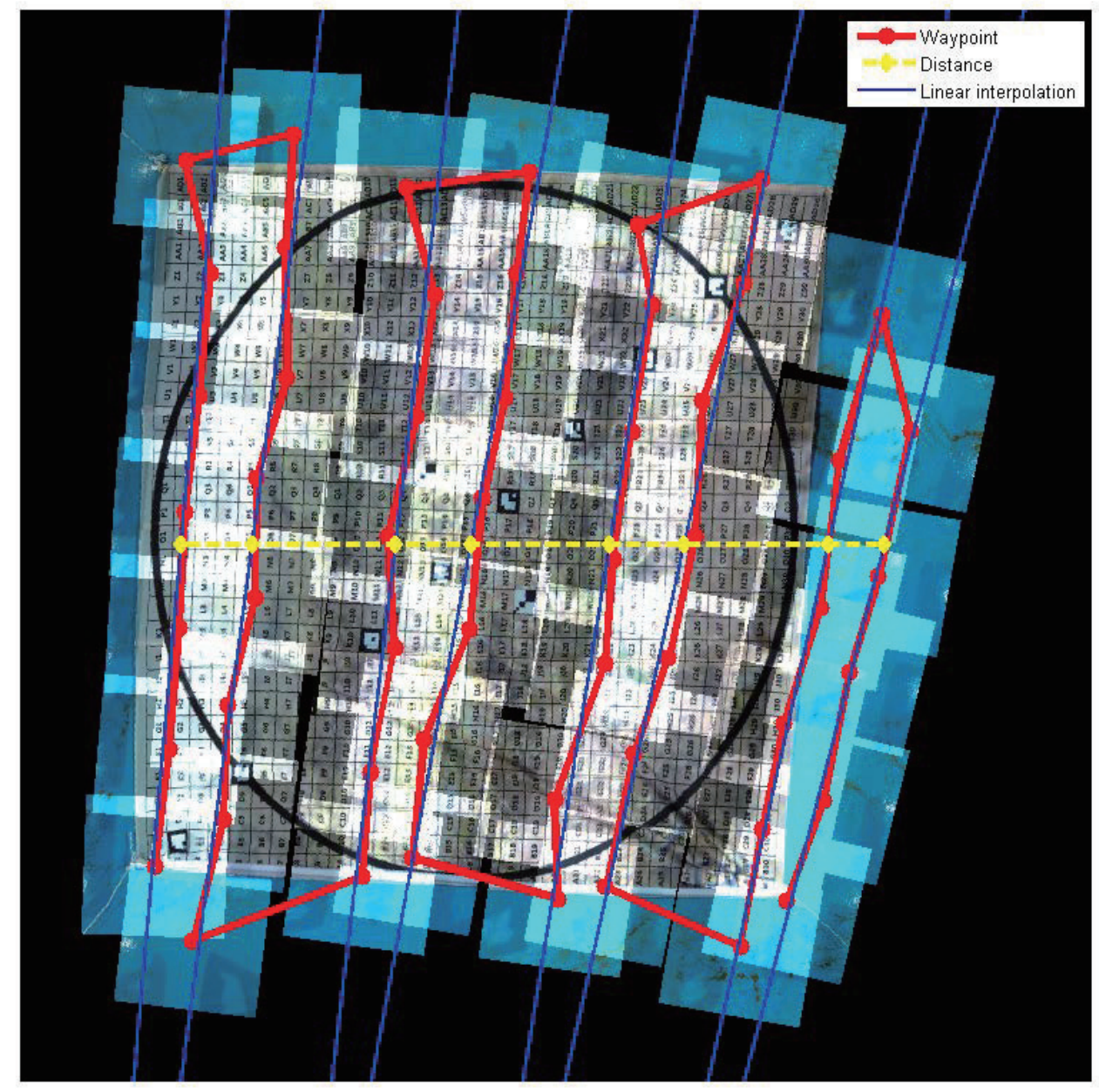

Figure 25: Source image arrangement in variable heading angle during scanning. 


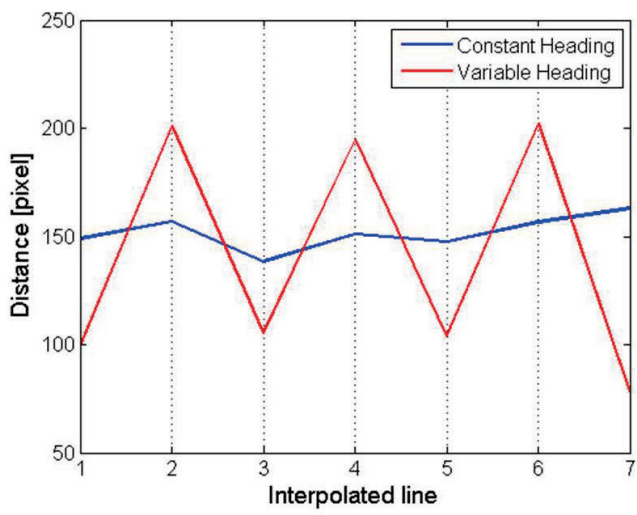

(a) Distance between adjacent lines

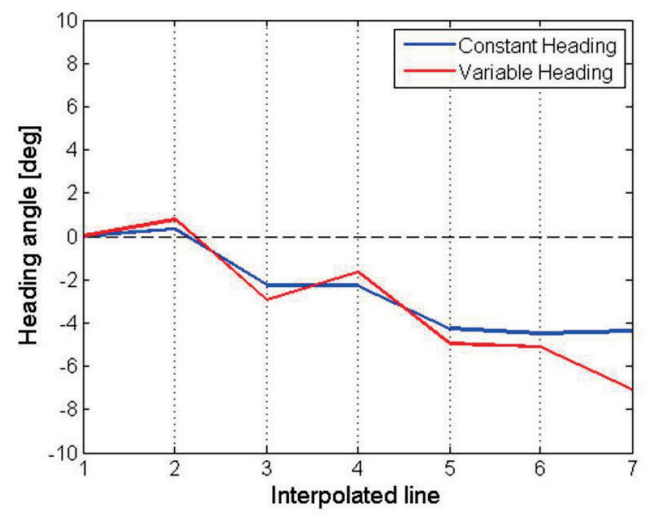

(b) Heading angle between adjacent lines

Figure 26: Distance and heading deviation between adjacent interpolated lines. 

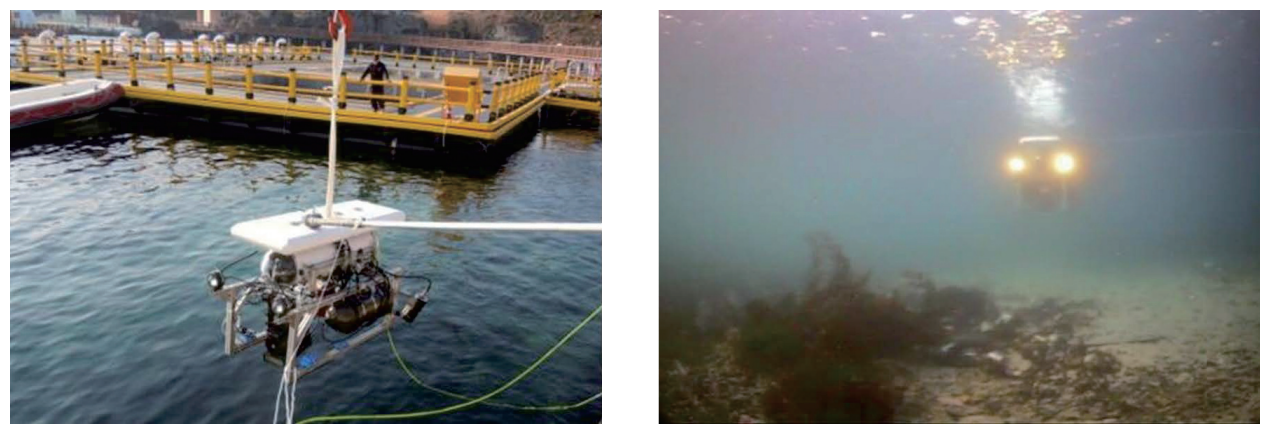

Figure 27: Overview of field trials in Yeongil bay. 


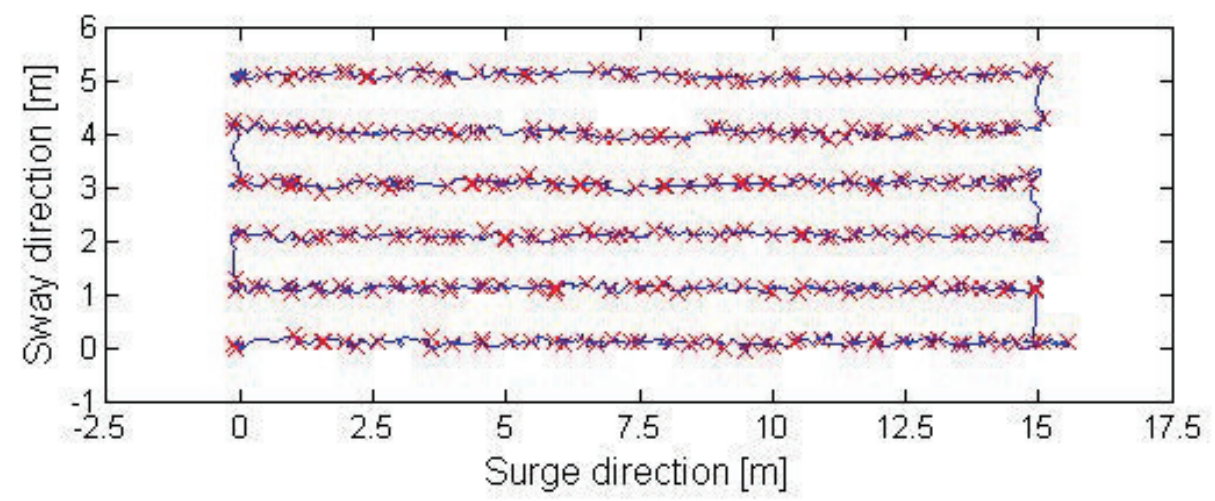

Figure 28: Trajectory of the Cyclops during the mosaicing mission. Blue line: trajectory. Cross marks: points where the images are captured. 


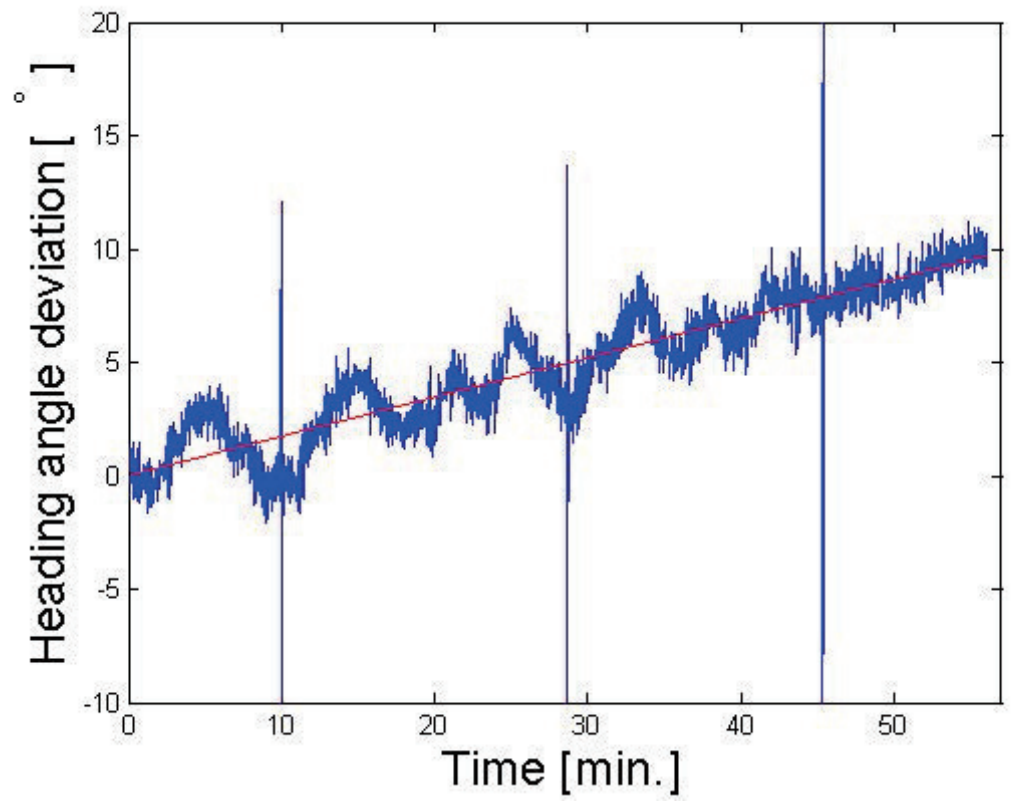

Figure 29: Heading angle deviation in field test. 


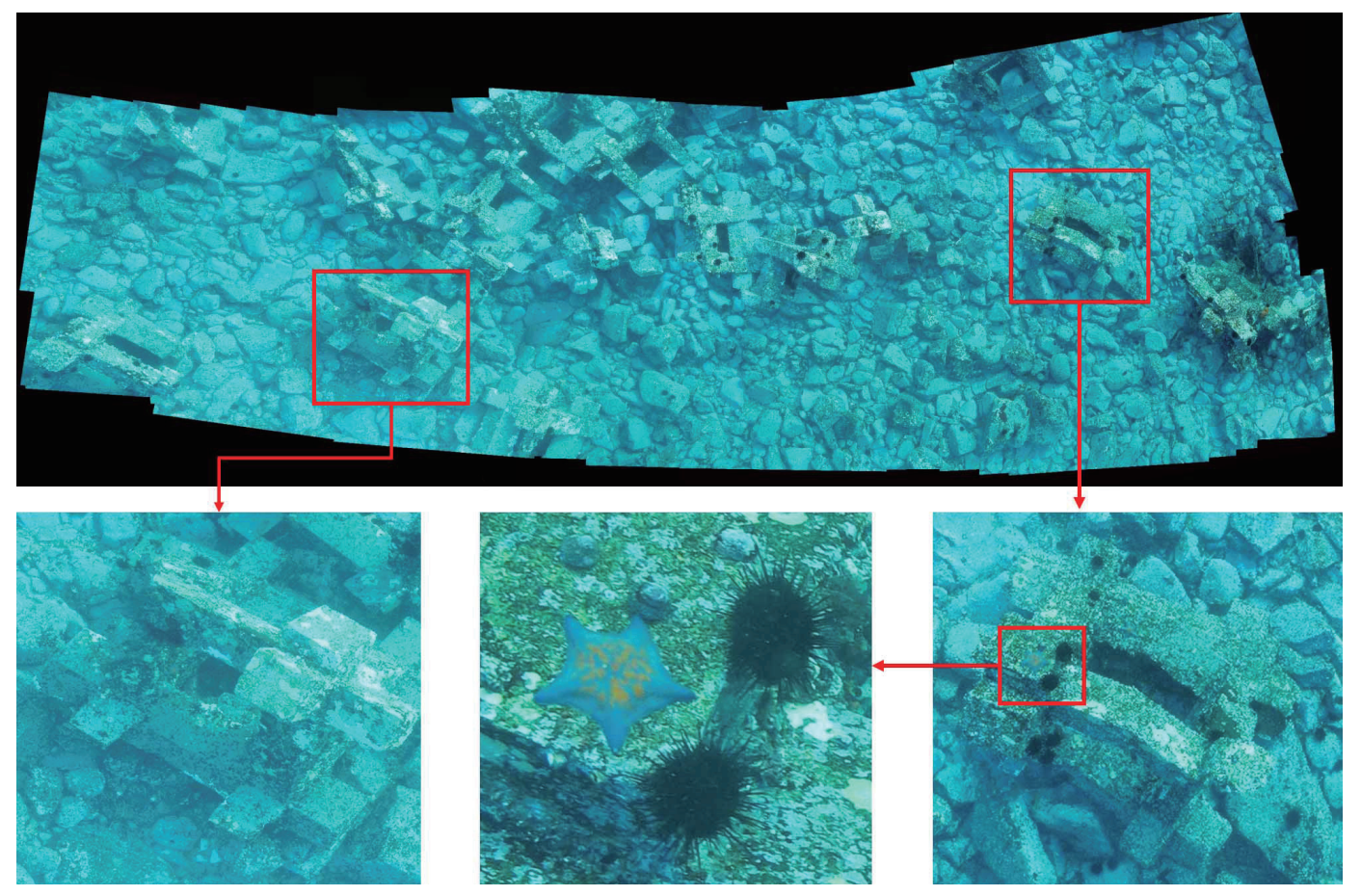

Figure 30: Image mosaicing results of field trials. 